\title{
ADMISSIBILITY IN FINITELY GENERATED QUASIVARIETIES *
}

\author{
GEORGE METCALFE AND CHRISTOPH RÖTHLISBERGER
}

Mathematics Institute, University of Bern, Sidlerstrasse 5, Bern 3012, Switzerland

e-mail address: \{george.metcalfe, christoph.roethlisberger\}@math.unibe.ch

\begin{abstract}
Checking the admissibility of quasiequations in a finitely generated (i.e., generated by a finite set of finite algebras) quasivariety $\mathcal{Q}$ amounts to checking validity in a suitable finite free algebra of the quasivariety, and is therefore decidable. However, since free algebras may be large even for small sets of small algebras and very few generators, this naive method for checking admissibility in $\mathcal{Q}$ is not computationally feasible. In this paper, algorithms are introduced that generate a minimal (with respect to a multiset well-ordering on their cardinalities) finite set of algebras such that the validity of a quasiequation in this set corresponds to admissibility of the quasiequation in $\mathcal{Q}$. In particular, structural completeness (validity and admissibility coincide) and almost structural completeness (validity and admissibility coincide for quasiequations with unifiable premises) can be checked. The algorithms are illustrated with a selection of well-known finitely generated quasivarieties, and adapted to handle also admissibility of rules in finite-valued logics.
\end{abstract}

\section{INTRODUCTION}

The problem of checking the validity of quasiequations in finitely generated (i.e., generated by a finite set of finite algebras) quasivarieties or, similarly, checking consequences from finite sets of formulas in finite-valued logics, is decidable and has been investigated extensively in the literature. In particular, uniform methods for generating proof systems to check validity such as tableaux, resolution, and multisequent calculi, have been developed, as have standard optimization techniques for these systems such as lemma generation and indexing (see, e.g., [21, 36, 2]). However, checking the admissibility of quasiequations in finitely generated quasivarieties, or similarly, checking the admissibility of rules in finite-valued logics, is not so well-understood. The problem is decidable, but a naive approach leads to computationally unfeasible procedures even for small sets of small algebras. The main goal of this paper is to define uniform methods that generate computationally acceptable proof systems for checking admissibility in an arbitrary finitely generated quasivariety.

2012 ACM CCS: [Theory of computation]: Models of computation; [Computing Methodologies]: Symbolic and algebraic manipulation-Symbolic and algebraic algorithms - Theorem proving algorithms.

Key words and phrases: Unification, Admissibility, Quasivariety, Finite Algebra, Free Algebra.

* A precursor to this paper, reporting preliminary results, has appeared as 32 .

The authors acknowledge support from Swiss National Science Foundation grants 20002_129507 and 200021_146748 and Marie Curie Reintegration Grant PIRG06-GA-2009-256492. 
Intuitively, a rule is said to be admissible in a logical system if it can be added to the system without producing any new theorems. More formally, a quasiequation is admissible in a class of algebras $\mathcal{K}$ if every $\mathcal{K}$-unifier of the premises is a $\mathcal{K}$-unifier of the conclusion, where a $\mathcal{K}$-unifier of an equation $\varphi \approx \psi$ is a substitution $\sigma$ such that $\sigma(\varphi) \approx \sigma(\psi)$ is valid in $\mathcal{K}$. Admissibility plays a fundamental meta-level role in describing "hidden properties" of classes of algebras and logical systems. For example, establishing the completeness of a logical system with respect to some restricted class of algebras (perhaps just one standard algebra) often involves showing that a certain rule or quasiequation is admissible; see, e.g., [30] for applications of the admissibility of rules in the context of fuzzy logics. Also, the closely related problem of deciding unifiability of concepts can be a useful tool for database redundancy checking in description logics [1. Moreover, it may be possible to automatically obtain admissible rules for classes of algebras and logics that can then be used to simplify reasoning steps or to speed up derivations for checking validity.

Admissibility (in tandem with unification) has been studied intensively in the context of intermediate and transitive modal logics and their algebras [35, 22, 17, 18, 25, 14, 4, 3, leading in some cases to proof systems for checking admissibility [19, 24, 23, 5]. Axiomatizations and characterizations have also been obtained for certain families of finite algebras and many-valued logics, in particular Łukasiewicz logics (or classes of MV-algebras) [26, 27] and other fuzzy logics [13, fragments of the substructural logic R-Mingle [29], and classes of De Morgan algebras [31, 11. However, a general theory, covering arbitrary finite algebras and finite-valued logics, has so far been lacking.

The starting point for this work is the observation (see Lemma 4.1.10 of [35] and Corollary 5.2 below) that for a finite set of finite algebras $\mathcal{K}$, admissibility in the quasivariety $\mathbb{Q}(\mathcal{K})$ amounts to validity in the free algebra on $n$ generators $\mathbf{F}_{\mathcal{K}}(n)$, where $n$ is the maximum cardinality of the algebras in $\mathcal{K}$. Since by Birkhoff's theorem on the structure of free algebras [8], this algebra $\mathbf{F}_{\mathcal{K}}(n)$ is finite, checking admissibility in $\mathbb{Q}(\mathcal{K})$ is decidable. On the other hand, even for small $n$ and a small set of small algebras $\mathcal{K}$, the size of $\mathbf{F}_{\mathcal{K}}(n)$ may be prohibitively large for checking validity. This is striking since validity and admissibility in $\mathbb{Q}(\mathcal{K})$ may coincide, $\mathbb{Q}(\mathcal{K})$ is then called structurally complete, or at least coincide for quasiequations with $\mathbb{Q}(\mathcal{K})$-unifiable premises, in which case, $\mathbb{Q}(\mathcal{K})$ is called almost structurally complete. In other cases, $\mathbb{Q}(\mathcal{K})$-admissibility may correspond to validity in other, often quite small, algebras. We provide general algorithms here that discover such algebras, or, more precisely, generate finite sets of finite algebras such that the $\mathbb{Q}(\mathcal{K})$-admissibility of a quasiequation corresponds to validity in the quasivariety generated by these algebras. It is shown, moreover, that these are the smallest sets of algebras with this property with respect to a standard well-ordering on the multiset of their cardinalities.

We proceed as follows. First, in Section 2, we recall some basic notions from universal algebra. Then in Section 3, we introduce some key ideas and methods for finitely generated quasivarieties; in particular, we apply a standard multiset well-ordering to the cardinalities of algebras in generating sets for quasivarieties, and provide an algorithm for finding the (unique up to isomorphism) minimal generating set of a finitely generated quasivariety. Section 4 provides characterizations of admissibility, unifiability, structural completeness, and almost structural completeness. These characterizations are then exploited in Section 5 to define corresponding algorithms, and illustrated using a selection of well-known finite algebras, confirming some known results from the literature, and establishing new ones. In Section 6, the approach is extended to finite-valued logics, where the designated values 
as well as the underlying finite algebra play a significant role. Finally, in Section 7, we conclude with some remarks on future directions for this research.

\section{Preliminaries}

Let us first recall some basic ideas from universal algebra, referring to [10, 20] for further details. Given an algebraic language $\mathcal{L}$ (i.e., without relation symbols), an $\mathcal{L}$-algebra $\mathbf{A}$ is an algebraic structure consisting of a set $A$ (the universe) and an $n$-ary function $\star^{\mathbf{A}}$ corresponding to each $n$-ary function symbol $\star$ of $\mathcal{L}$ (as usual, calling nullary functions constants). We call $\mathbf{A}$ finite if $A$ is a finite set and $\mathcal{L}$ consists of finitely many function symbols. A congruence on an $\mathcal{L}$-algebra $\mathbf{A}$ is an equivalence relation $\theta$ on $A$ satisfying for each $n$-ary function symbol $\star$ of $\mathcal{L}:\left\{\left(a_{1}, b_{1}\right), \ldots,\left(a_{n}, b_{n}\right)\right\} \subseteq \theta$ implies $\left(\star^{\mathbf{A}}\left(a_{1}, \ldots, a_{n}\right), \star^{\mathbf{A}}\left(b_{1}, \ldots, b_{n}\right)\right) \in \theta$. The congruences of $\mathbf{A}$ form a complete lattice $\operatorname{Con}(\mathbf{A})$ with bottom element $\Delta_{\mathbf{A}}=\{(a, a): a \in A\}$ and top element $\nabla_{\mathbf{A}}=\{(a, b): a, b \in A\}$, where the meet of a set of congruences on $\mathbf{A}$ is just the intersection of those congruences. Given $\theta \in \operatorname{Con}(\mathbf{A})$, the quotient algebra of $\mathbf{A}$ by $\theta$ is the $\mathcal{L}$-algebra $\mathbf{A} / \theta$ with universe $A / \theta$ consisting of the equivalence classes $a / \theta$ for $a \in A$ with functions defined for each $n$-ary function symbol $\star$ of $\mathcal{L}$ by $\star^{\mathbf{A} / \theta}\left(a_{1} / \theta, \ldots, a_{n} / \theta\right)=\star^{\mathbf{A}}\left(a_{1}, \ldots, a_{n}\right) / \theta$.

Term algebras $\operatorname{Tm}_{\mathcal{L}}(X)$ are defined over a set of variables $X$ in the usual way, writing just $\mathbf{T m}_{\mathcal{L}}$ when $X$ is a fixed countably infinite set, and letting $\varphi, \psi$ stand for arbitrary members of the universe $\operatorname{Tm}_{\mathcal{L}}$ called $\mathcal{L}$-terms. An $\mathcal{L}$-equation is an ordered pair of $\mathcal{L}$-terms, written $\varphi \approx \psi$. An $\mathcal{L}$-clause is defined as an ordered pair $\Sigma, \Delta$ of finite sets of $\mathcal{L}$-equations, written $\Sigma \Rightarrow \Delta$, and called an $\mathcal{L}$-quasiequation if $|\Delta|=1$ and an $\mathcal{L}$-negative clause if $\Delta=\emptyset$. As usual, if the language is clear from the context, we may omit the prefix $\mathcal{L}$.

Let us fix $\mathcal{K}$ to be a class of $\mathcal{L}$-algebras, noting that often in what follows $\mathcal{K}$ will consist of a finite set of $\mathcal{L}$-algebras $\mathbf{A}_{1}, \ldots, \mathbf{A}_{n}$, and in this case we typically omit brackets. Given a finite set of $\mathcal{L}$-equations $\Sigma \cup \Delta$, we write $\Sigma \models \mathcal{K} \Delta$ and say that the $\mathcal{L}$-clause $\Sigma \Rightarrow \Delta$ is $\mathcal{K}$-valid, if for every $\mathbf{A} \in \mathcal{K}$ and homomorphism $h: \mathbf{T m}_{\mathcal{L}} \rightarrow \mathbf{A}$,

$$
\Sigma \subseteq \operatorname{ker} h \quad \text { implies } \quad \Delta \cap \operatorname{ker} h \neq \emptyset,
$$

recalling that $\operatorname{ker} h=\{(\varphi, \psi): h(\varphi)=h(\psi)\}$. We also say that $\Sigma$ is $\mathcal{K}$-satisfiable if $\Sigma \subseteq \operatorname{ker} h$ for some $\mathbf{A} \in \mathcal{K}$ and homomorphism $h: \mathbf{T m}_{\mathcal{L}} \rightarrow \mathbf{A}$.

The class $\mathcal{K}$ is said to be axiomatized by a set of $\mathcal{L}$-clauses $\Lambda$ if $\mathcal{K}$ is the class of $\mathcal{L}$ algebras $\mathbf{A}$ such that all $\mathcal{L}$-clauses in $\Lambda$ are $\mathbf{A}$-valid. $\mathcal{K}$ is called a variety, quasivariety, or antivariety if it is axiomatized by a set of $\mathcal{L}$-equations, $\mathcal{L}$-quasiequations, or $\mathcal{L}$-negative clauses, respectively. The variety $\mathbb{V}(\mathcal{K})$, quasivariety $\mathbb{Q}(\mathcal{K})$, and antivariety $\mathbb{V}^{-}(\mathcal{K})$ generated by $\mathcal{K}$ are the smallest variety, quasivariety, and antivariety containing $\mathcal{K}$, respectively.

Moreover, let $\mathbb{H}, \mathbb{I}, \mathbb{S}, \mathbb{P}, \mathbb{P}_{U}, \mathbb{P}_{U}^{*}$, and $\mathbb{H}^{-1}$ be the class operators of taking homomorphic images, isomorphic images, subalgebras, products, ultraproducts, non-empty ultraproducts, and homomorphic preimages, respectively. Then $\mathbb{V}(\mathcal{K})=\mathbb{H} \mathbb{S P}(\mathcal{K}), \mathbb{Q}(\mathcal{K})=\mathbb{I S P P} \mathbb{P}_{U}(\mathcal{K})$, and $\mathbb{V}^{-}(\mathcal{K})=\mathbb{H}^{-1} \mathbb{S P}_{U}^{*}(\mathcal{K})$, and if $\mathcal{K}$ is a finite set of finite algebras, these last two equivalences refine to $\mathbb{Q}(\mathcal{K})=\mathbb{I S P}(\mathcal{K})$ and $\mathbb{V}^{-}(\mathcal{K})=\mathbb{H}^{-1} \mathbb{S}(\mathcal{K})$ (see [10, 20] for details).

Given a language $\mathcal{L}$ and a set of variables $X$ such that either $X \neq \emptyset$ or $\mathcal{L}$ contains at least one constant symbol, the term algebra $\operatorname{Tm}_{\mathcal{L}}(X)$ exists and admits a congruence:

$$
\theta_{\mathcal{K}}(X)=\bigcap\left\{\phi \in \operatorname{Con}\left(\mathbf{T m}_{\mathcal{L}}(X)\right): \mathbf{T}_{\mathcal{L}}(X) / \phi \in \mathbb{I S}(\mathcal{K})\right\}
$$


Following [10], we let $\bar{X}=X / \theta_{\mathcal{K}}(X)$ and define the free algebra of $\mathcal{K}$ over $\bar{X}$ :

$$
\mathbf{F}_{\mathcal{K}}(\bar{X})=\mathbf{T}_{\mathcal{L}}(X) / \theta_{\mathcal{K}}(X)
$$

Then $\mathbf{F}_{\mathcal{K}}(\bar{X})$ has the universal mapping property for $\mathcal{K}$ over $\bar{X}$ : namely, for each $\mathbf{A} \in \mathcal{K}$, any map from $\bar{X}$ to $A$ extends to a homomorphism from $\mathbf{F}_{\mathcal{K}}(\bar{X})$ to $\mathbf{A}$ ([10, Theorem II.10.10]). If also $\mathcal{K} \neq \emptyset$, then $\mathbf{F}_{\mathcal{K}}(\bar{X}) \in \mathbb{I S P}(\mathcal{K}) \subseteq \mathbb{Q}(\mathcal{K})([10$, Theorem II.10.12]).

Note that $\mathbf{F}_{\mathcal{K}}(\bar{X}) \cong \mathbf{F}_{\mathcal{K}}(\bar{Y})$ whenever $|X|=|Y|$ (also $|\bar{X}|=|X|$ if $\mathcal{K}$ contains at least one non-trivial algebra). Hence we may consider for each cardinal $\kappa$, the (unique up to isomorphism) free algebra of $\mathcal{K}$ on $\kappa$ generators $\mathbf{F}_{\mathcal{K}}(\kappa)$, where $\mathbf{F}_{\mathcal{K}}\left(\kappa_{1}\right)$ is a subalgebra of $\mathbf{F}_{\mathcal{K}}\left(\kappa_{2}\right)$ for cardinals $\kappa_{1} \leq \kappa_{2}$. We note also that $\mathbb{V}(\mathcal{K})=\mathbb{V}\left(\mathbf{F}_{\mathcal{K}}(\omega)\right)$ and that $\mathbb{V}\left(\mathcal{K}_{1}\right)=\mathbb{V}\left(\mathcal{K}_{2}\right)$ implies $\mathbf{F}_{\mathcal{K}_{1}}(\omega)=\mathbf{F}_{\mathcal{K}_{2}}(\omega)$ (see [10, Corollary II.11.10; Exercise II.11.2]).

\section{Finitely Generated Quasivarieties}

A quasivariety $\mathcal{Q}$ is said to be finitely generated if $\mathcal{Q}=\mathbb{Q}(\mathcal{K})$ for some finite (generating) set $\mathcal{K}$ of finite $\mathcal{L}$-algebras. Our first goal in this section will be to define a reasonable measure for comparing these generating sets; we will then describe a method for obtaining a "smallest" generating set according to this measure. More precisely, we apply the standard multiset well-ordering defined by Dershowitz and Manna in [16] to the multiset of the cardinalities of algebras in $\mathcal{K}$. We show that by decomposing finite $\mathcal{L}$-algebras into their $\mathcal{Q}$-subdirectly irreducible components and appropriately refining the set of algebras obtained, we arrive at a (unique up to isomorphism) minimal set of $\mathcal{L}$-algebras that still generates $\mathcal{Q}$.

Recall that a multiset over a set $S$ is an ordered pair $\langle S, f\rangle$ where $f$ is a function $f: S \rightarrow \mathbb{N}$, and is called finite if $\{x \in S: f(x)>0\}$ is finite. For a well-ordered set $\langle S, \leq\rangle$, the multiset ordering $\leq_{m}$ on the set $M(S)$ of finite multisets over $S$ is defined by $\langle S, f\rangle \leq_{m}\langle S, g\rangle$ if $f(x)>g(x)$ implies that for some $y \in S, y>x$ and $g(y)>f(y)$. It then follows that $\leq_{m}$ is a well-ordering of $M(S)$ (see [16]). As usual, we write a finite multiset of elements from $S$ as $\left[a_{1}, \ldots, a_{n}\right]$ where $a_{1}, \ldots, a_{n} \in S$ may include repetitions.

A set of finite $\mathcal{L}$-algebras $\left\{\mathbf{A}_{1}, \ldots, \mathbf{A}_{n}\right\}$ will be called a minimal generating set for the quasivariety $\mathbb{Q}\left(\mathbf{A}_{1}, \ldots, \mathbf{A}_{n}\right)$ if for every set of finite $\mathcal{L}$-algebras $\left\{\mathbf{B}_{1}, \ldots, \mathbf{B}_{k}\right\}$ :

$$
\mathbb{Q}\left(\mathbf{A}_{1}, \ldots, \mathbf{A}_{n}\right)=\mathbb{Q}\left(\mathbf{B}_{1}, \ldots, \mathbf{B}_{k}\right) \quad \text { implies } \quad\left[\left|A_{1}\right|, \ldots,\left|A_{n}\right|\right] \leq_{m}\left[\left|B_{1}\right|, \ldots,\left|B_{k}\right|\right] .
$$

There are of course many measures that could be used to compare generating sets. Indeed, it may seem unreasonable to view one hundred algebras with three elements as an improvement on a single algebra with four elements. However, the order is optimal in the following sense. In general, checking a quasiequation with $r$ variables in a finite algebra $\mathbf{A}$ requires checking $|A|^{r}$ assignments of variables to elements of $\mathbf{A}$. But then checking validity in $\left\{\mathbf{A}_{1}, \ldots, \mathbf{A}_{n}\right\}$ will involve checking fewer assignments of variables than checking validity in $\left\{\mathbf{B}_{1}, \ldots, \mathbf{B}_{k}\right\}$ if $\left[\left|A_{1}\right|, \ldots,\left|A_{n}\right|\right] \leq_{m}\left[\left|B_{1}\right|, \ldots,\left|B_{k}\right|\right]$ for quasiequations with sufficiently many variables.

To obtain minimal generating sets for $\mathbb{Q}(\mathcal{K})$ where $\mathcal{K}$ is a finite set of finite algebras, we consider representations of the algebras in $\mathcal{K}$ using smaller algebras of $\mathbb{Q}(\mathcal{K})$. An algebra $\mathbf{A}$ is called a subdirect product of algebras $\mathbf{A}_{1}, \ldots, \mathbf{A}_{n}$ if there exist surjective homomorphisms $f_{i}: \mathbf{A} \rightarrow \mathbf{A}_{i}$ for $i=1 \ldots n$ such that the induced homomorphism

$$
f: \mathbf{A} \rightarrow \mathbf{A}_{1} \times \cdots \times \mathbf{A}_{n}, \quad f(x)=\left\langle f_{1}(x), \ldots, f_{n}(x)\right\rangle
$$

is an embedding. In this case, $f$ is called a subdirect representation of $\mathbf{A}$ and $\mathbf{A}_{1}, \ldots, \mathbf{A}_{n}$ are called subdirect components (for this representation) of $\mathbf{A}$. If $\mathcal{Q}$ is a quasivariety and 
$\mathbf{A}_{1}, \ldots, \mathbf{A}_{n} \in \mathcal{Q}$, then $\mathbf{A}$ is called a $\mathcal{Q}$-subdirect product of $\mathbf{A}_{1}, \ldots, \mathbf{A}_{n}$ and $f$ is called a $\mathcal{Q}$ subdirect embedding. $\mathbf{A}$ is called $\mathcal{Q}$-subdirectly irreducible if for every $\mathcal{Q}$-subdirect embedding $f: \mathbf{A} \rightarrow \mathbf{A}_{1} \times \cdots \times \mathbf{A}_{n}, \mathbf{A}$ is isomorphic to $\mathbf{A}_{i}$ for some $i \in\{1, \ldots, n\}$.

We will make essential use of the following:

Lemma 3.1 ([12, Corollary 6]). Let $\mathcal{Q}$ be a quasivariety and $\mathbf{A} \in \mathcal{Q}$. Then $\mathbf{A}$ is a $\mathcal{Q}$ subdirect product of $\mathcal{Q}$-subdirectly irreducible members of $\mathcal{Q}$.

Lemma 3.2 ([20, Proposition 3.1.6]). If $\mathcal{Q}=\mathbb{Q}(\mathcal{K})$ for a finite set $\mathcal{K}$ of finite algebras and $\mathbf{A}$ is a $\mathcal{Q}$-subdirectly irreducible algebra, then $\mathbf{A} \in \mathbb{I S}(\mathcal{K})$.

Observe now that if $\mathcal{Q}$ is a quasivariety and $\mathbf{A} \in \mathcal{Q}$ is a $\mathcal{Q}$-subdirect product of $\mathbf{A}_{1}, \ldots, \mathbf{A}_{n}$, then each $\mathbf{A}_{i}$ is in $\mathcal{Q}$ by definition, and $\mathbf{A}$ is isomorphic to a subalgebra of a product of members of $\mathcal{Q}$. Hence we obtain:

Lemma 3.3. Let $\mathcal{K}$ be a class of $\mathcal{L}$-algebras and suppose that $\mathcal{K}^{\prime}$ is obtained from $\mathcal{K}$ by either (a) replacing $\mathbf{A} \in \mathcal{K}$ with $\mathbf{A}_{1}, \ldots, \mathbf{A}_{n}$ where $\mathbf{A}$ is a $\mathbb{Q}(\mathcal{K})$-subdirect product of $\mathbf{A}_{1}, \ldots, \mathbf{A}_{n}$, or (b) replacing $\mathbf{A}, \mathbf{B} \in \mathcal{K}$ with $\mathbf{B}$ where $\mathbf{A} \in \mathbb{I S}(\mathbf{B})$. Then $\mathbb{Q}(\mathcal{K})=\mathbb{Q}\left(\mathcal{K}^{\prime}\right)$.

In particular, replacing each algebra $\mathbf{A}$ in a finite set $\mathcal{K}$ of finite algebras with the $\mathbb{Q}(\mathcal{K})$ subdirectly irreducible algebras in some $\mathbb{Q}(\mathcal{K})$-subdirect representation of $\mathbf{A}$, then removing any algebra that embeds into another algebra in the set, produces a minimal generating set for $\mathbb{Q}(\mathcal{K})$ that is unique up to isomorphism.

Theorem 3.4. Suppose that $\mathcal{Q}=\mathbb{Q}\left(\mathbf{A}_{1}, \ldots, \mathbf{A}_{n}\right)$ where $\mathbf{A}_{i}$ is a finite $\mathcal{Q}$-subdirectly irreducible algebra for $i \in\{1 \ldots n\}$ and $\mathbf{A}_{i} \notin \mathbb{I S}\left(\mathbf{A}_{j}\right)$ for $j \neq i$. Then $\left\{\mathbf{A}_{1}, \ldots, \mathbf{A}_{n}\right\}$ is the unique minimal generating set for $\mathcal{Q}$ up to isomorphism.

Proof. Let $\mathcal{Q}=\mathbb{Q}\left(\mathbf{A}_{1}, \ldots, \mathbf{A}_{n}\right)$ where $\mathbf{A}_{i}$ is a finite $\mathcal{Q}$-subdirectly irreducible algebra for $i \in\{1 \ldots n\}$ and $\mathbf{A}_{i} \notin \mathbb{I S}\left(\mathbf{A}_{j}\right)$ for $j \neq i$. Suppose for a contradiction that $\mathcal{Q}=\mathbb{Q}\left(\mathbf{B}_{1}, \ldots, \mathbf{B}_{k}\right)$ and $\left[\left|B_{1}\right|, \ldots,\left|B_{k}\right|\right]<_{m}\left[\left|A_{1}\right|, \ldots,\left|A_{n}\right|\right]$. Without loss of generality, we can suppose that $\mathbf{B}_{j}$ is $\mathcal{Q}$-subdirectly irreducible for $j \in\{1, \ldots, k\}$; otherwise, by Lemmas 3.1 and 3.3 , $\mathbf{B}_{j}$ can be replaced with the $\mathcal{Q}$-subdirectly irreducible components of a $\mathcal{Q}$-subdirect representation of $\mathbf{B}_{j}$ and we obtain a smaller (according to $\leq_{m}$ ) generating set of algebras for $\mathcal{Q}$.

It follows that there exists a largest $r \in \mathbb{N}$ such that there are strictly more occurrences of $r$ in $\left[\left|A_{1}\right|, \ldots,\left|A_{n}\right|\right]$ than in $\left[\left|B_{1}\right|, \ldots,\left|B_{k}\right|\right]$, and for each $r^{\prime}>r$, the number of occurrences of $r^{\prime}$ in $\left[\left|A_{1}\right|, \ldots,\left|A_{n}\right|\right]$ and $\left[\left|B_{1}\right|, \ldots,\left|B_{k}\right|\right]$ are equal. Each $\mathbf{A}_{i}$ is finite and $\mathcal{Q}$-subdirectly irreducible, and hence by Lemma 3.2, embeds into some $\mathbf{B}_{j}$ where $\left|A_{i}\right| \leq\left|B_{j}\right|$. If every $\mathbf{A}_{i}$ of size $r$ embeds into, and is hence isomorphic to, a $\mathbf{B}_{j}$ of size $r$, then (by the pigeonhole principle) there must be two isomorphic algebras in $\left\{\mathbf{A}_{1}, \ldots, \mathbf{A}_{n}\right\}$, a contradiction. Hence, suppose without loss of generality that $\mathbf{A}_{1}$ embeds into $\mathbf{B}_{1}$ with $\left|A_{1}\right|=r$ and $\left|B_{1}\right|>r$. But notice now that $\mathbf{B}_{1}$ is also $\mathcal{Q}$-subdirectly irreducible and hence embeds into some $\mathbf{A}_{i}$ with $i \in\{2, \ldots, n\}$. So $\mathbf{A}_{1} \in \mathbb{I S}\left(\mathbf{A}_{i}\right)$, a contradiction.

Finally, consider any minimal generating set $\left\{\mathbf{B}_{1}, \ldots, \mathbf{B}_{k}\right\}$ for $\mathcal{Q}$, and suppose for a contradiction that $\mathbf{B}_{i} \notin \mathbb{I}\left(\mathbf{A}_{1}, \ldots, \mathbf{A}_{n}\right)$ for some $i \in\{1, \ldots, k\}$. Then by Lemma 3.2, $\mathbf{B}_{i}$ properly embeds into $\mathbf{A}_{j}$ for some $j \in\{1, \ldots, n\}$. But also by Lemma 3.2, $\mathbf{A}_{j}$ embeds into $\mathbf{B}_{d}$ for some $d \in\{1, \ldots, k\} \backslash\{i\}$. It follows that $\mathbf{B}_{i}$ can be embedded into the strictly larger algebra $\mathbf{B}_{d}$. But then $\left\{\mathbf{B}_{1}, \ldots, \mathbf{B}_{k}\right\}$ is not a minimal generating set for $\mathcal{Q}$, a contradiction.

In the remainder of this section, we develop some results for $\mathcal{Q}$-subdirect products and $\mathcal{Q}$-subdirectly irreducible algebras, and present an algorithm for obtaining minimal 
generating sets for finitely generated quasivarieties. First, let us recall the following theorem of Birkhoff, which establishes a useful relationship between subdirect representations of a given algebra $\mathbf{A}$ and sets of congruences on $\mathbf{A}$.

Lemma 3.5 ([9, Universal Algebra, Theorem 11]). If $\mathbf{A}$ is a subdirect product of the family $\left(\mathbf{A}_{i}\right)_{i \in I}$, then there exist for $i \in I$, congruences $\theta_{i} \in \operatorname{Con}(\mathbf{A})$ such that $\mathbf{A}_{i} \cong \mathbf{A} / \theta_{i}$ and $\bigcap_{i \in I} \theta_{i}=\Delta_{\mathbf{A}}$. Conversely, for any family of congruences $\left(\theta_{i}\right)_{i \in I}$ on $\mathbf{A}$, the quotient $\mathbf{A} /\left(\bigcap_{i \in I} \theta_{i}\right)$ is a subdirect product of the family $\left(\mathbf{A} / \theta_{i}\right)_{i \in I}$.

The set of $\mathcal{Q}$-congruences on $\mathbf{A}$ is defined as $\operatorname{Con}_{\mathcal{Q}}(\mathbf{A})=\{\theta \in \operatorname{Con}(\mathbf{A}): \mathbf{A} / \theta \in \mathcal{Q}\}$. Clearly, the above lemma also holds for $\mathcal{Q}$-subdirect representations of an algebra $\mathbf{A}$ with respect to $\mathcal{Q}$-congruences. Note, moreover, that the number of congruences needed to obtain a subdirect representation of a finite algebra $\mathbf{A}$ is at most $|A|$, the maximal number of coatoms of the congruence lattice $\operatorname{Con}(\mathbf{A})$.

Corollary 3.6. Let $\mathcal{Q}$ be a quasivariety and $\mathbf{A} \in \mathcal{Q}$.

(a) If $\mathbf{A}$ is a $\mathcal{Q}$-subdirect product of the family $\left(\mathbf{A}_{i}\right)_{i \in I}$, then there exist for $i \in I$, $\mathcal{Q}$ congruences $\theta_{i} \in \operatorname{Con}_{\mathcal{Q}}(\mathbf{A})$ such that $\mathbf{A}_{i} \cong \mathbf{A} / \theta_{i}$ and $\bigcap_{i \in I} \theta_{i}=\Delta_{\mathbf{A}}$. Conversely, for any family of $\mathcal{Q}$-congruences $\left(\theta_{i}\right)_{i \in I}$ on $\mathbf{A}$ with $\bigcap_{i \in I} \theta_{i}=\Delta_{\mathbf{A}}, \mathbf{A}$ is a $\mathcal{Q}$-subdirect product of the family $\left(\mathbf{A} / \theta_{i}\right)_{i \in I}$.

(b) $\mathbf{A}$ is $\mathcal{Q}$-subdirectly irreducible iff the bottom element $\Delta_{\mathbf{A}}$ of $\mathrm{Con}_{\mathcal{Q}}(\mathbf{A})$ is meet-irreducible (i.e., if $\bigcap_{i \in I} \theta_{i}=\Delta_{\mathbf{A}}$ for $\theta_{i} \in \operatorname{Con}_{\mathcal{Q}}(\mathbf{A})$, then $\Delta_{\mathbf{A}}=\theta_{i}$ for some $i \in I$ ).

The problem of finding the congruence closure for a given equivalence relation on a finite algebra, i.e., the smallest congruence containing this equivalence, can be solved in polynomial time. This result was used in [15] to provide a polynomial time algorithm for calculating a subdirect representation of a finite algebra. The problem of finding the $\mathcal{Q}$-congruence closure of an equivalence relation on a finite algebra with respect to a finitely generated quasivariety $\mathcal{Q}$ appears to be much harder, however. Instead, we use here the following characterization of $\mathcal{Q}$-subdirectly irreducible algebras as the basis for a procedure that constructs $\mathcal{Q}$-subdirectly irreducible components for a $\mathcal{Q}$-subdirect representation of a given finite algebra without needing to calculate the $\mathcal{Q}$-congruence lattice.

Lemma 3.7. For a finite set $\mathcal{K}$ of finite algebras and $\mathbf{A} \in \mathbb{Q}(\mathcal{K})$, the following are equivalent:

(1) $\mathbf{A}$ is $\mathbb{Q}(\mathcal{K})$-subdirectly irreducible.

(2) $\bigcap\left\{\theta \in \operatorname{Con}(\mathbf{A}) \backslash\left\{\Delta_{\mathbf{A}}\right\}: \mathbf{A} / \theta \in \mathbb{I S}(\mathcal{K})\right\} \neq \Delta_{\mathbf{A}}$.

Proof. For convenience, let

$$
\Theta=\left\{\theta \in \operatorname{Con}(\mathbf{A}) \backslash\left\{\Delta_{\mathbf{A}}\right\}: \mathbf{A} / \theta \in \mathbb{I} S(\mathcal{K})\right\} \subseteq \operatorname{Con}_{\mathbb{Q}(\mathcal{K})}(\mathbf{A}) .
$$

$(1) \Rightarrow(2)$ We proceed contrapositively. If $\bigcap \Theta=\Delta_{\mathbf{A}}$, then by Corollary 3.6(a), $\mathbf{A}$ is a $\mathbb{Q}(\mathcal{K})$-subdirect product of algebras in $\{\mathbf{A} / \theta: \theta \in \Theta\}$. But also by Corollary 3.6(b), since $\Delta_{\mathbf{A}} \notin \Theta, \mathbf{A}$ is not $\mathbb{Q}(\mathcal{K})$-subdirectly irreducible.

$(2) \Rightarrow(1)$ Again, we proceed contrapositively. If $\mathbf{A}$ is not $\mathbb{Q}(\mathcal{K})$-subdirectly irreducible, then combining Lemma 3.1 and Corollary 3.6. there exist $\left(\theta_{i}\right)_{i \in I} \subseteq \operatorname{Con}_{\mathbb{Q}(\mathcal{K})}(\mathbf{A}) \backslash\left\{\Delta_{\mathbf{A}}\right\}$ such that $\bigcap_{i \in I} \theta_{i}=\Delta_{\mathbf{A}}$ and $\mathbf{A}$ is a $\mathbb{Q}(\mathcal{K})$-subdirect product of $\mathbb{Q}(\mathcal{K})$-subdirectly irreducible algebras $\mathbf{A} / \theta_{i}(i \in I)$. But then also by Lemma 3.2, we have $\mathbf{A} / \theta_{i} \in \mathbb{I S}(\mathcal{K})$ for each $i \in I$. So $\left(\theta_{i}\right)_{i \in I} \subseteq \Theta$, and hence $\bigcap \Theta=\Delta_{\mathbf{A}}$. 


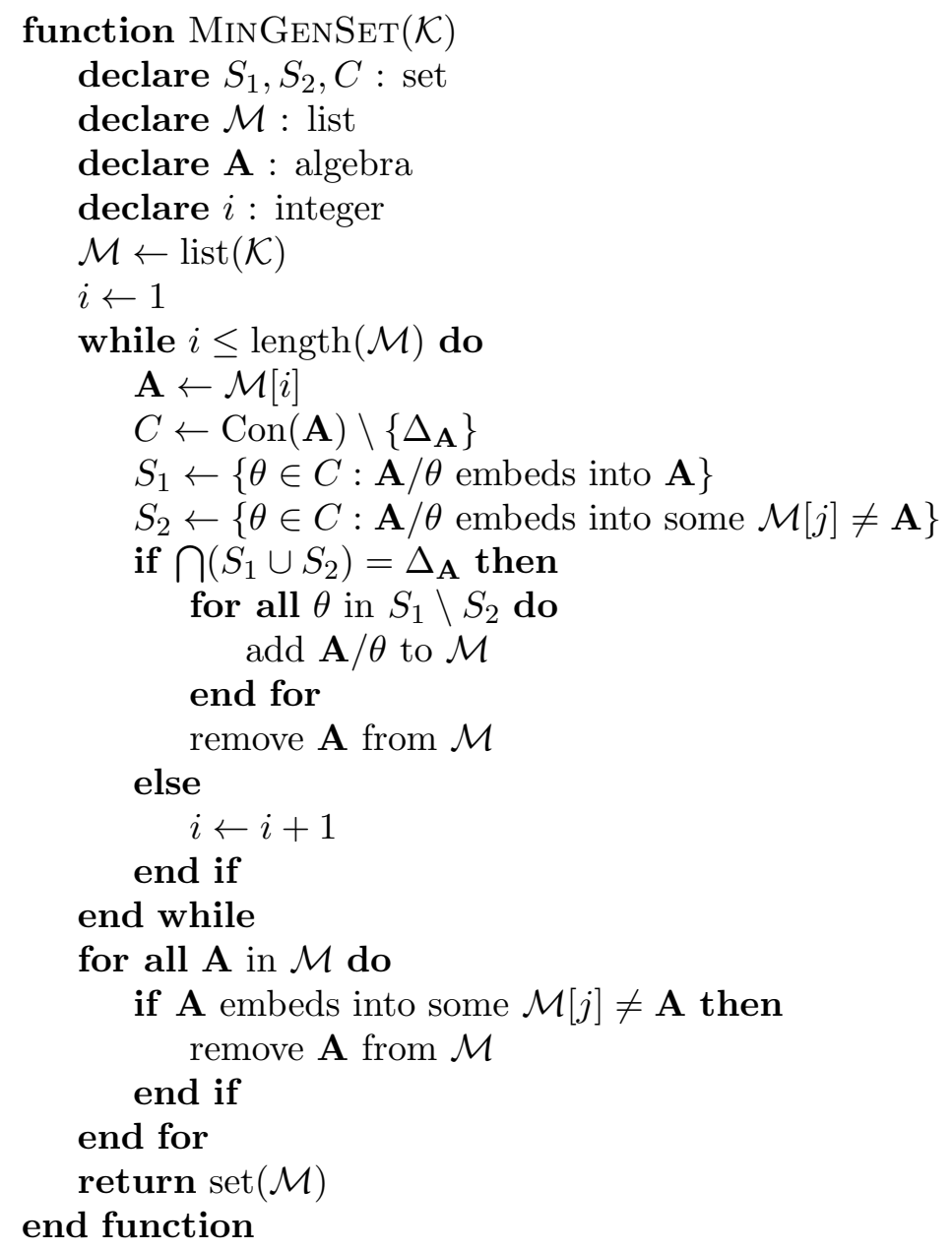

Figure 1: For a finite set $\mathcal{K}$ of finite algebras, return the minimal generating set of $\mathbb{Q}(\mathcal{K})$.

We now have all the ingredients necessary to describe an algorithm MinGenSet (see Figure 1) that calculates the (unique up to isomorphism) minimal generating set for a quasivariety $\mathcal{Q}=\mathbb{Q}(\mathcal{K})$, where $\mathcal{K}$ is a finite set of finite $\mathcal{L}$-algebras. By Theorem 3.4, it suffices to find a set of $\mathcal{Q}$-subdirectly irreducible algebras that generates $\mathcal{Q}$, where no member of the set embeds into another member of the set.

The algorithm proceeds by considering each $\mathbf{A} \in \mathcal{K}$ in turn. First, the congruence lattice $\operatorname{Con}(\mathbf{A})$ is generated (line 10) by checking for all equivalence relations if they are congruences. Next, the congruences $\theta \in \operatorname{Con}(\mathbf{A}) \backslash\left\{\Delta_{\mathbf{A}}\right\}$ such that $\mathbf{A} / \theta$ embeds into $\mathbf{A}$ or some other member of $\mathcal{K}$ are collected in sets $S_{1}$ and $S_{2}$, respectively. If $\bigcap\left(S_{1} \cup S_{2}\right) \neq \Delta_{\mathbf{A}}$, then $\mathbf{A}$ is $\mathcal{Q}$-subdirectly irreducible by Lemma 3.7, so the algorithm proceeds to the next algebra in $\mathcal{K}$. Otherwise $\bigcap\left(S_{1} \cup S_{2}\right)=\Delta_{\mathbf{A}}$ and by Lemma 3.7, A is not $\mathcal{Q}$-subdirectly irreducible. In this case, for each $\theta \in S_{1} \backslash S_{2}$, the algebra $\mathbf{A} / \theta$ is added to $\mathcal{K}$ (line 15) and $\mathbf{A}$ is removed from $\mathcal{K}$ (line 17). Note that since the cardinalities of the added algebras are strictly smaller than the cardinality of the removed algebra, the new set of algebras is smaller according to the multiset ordering defined in Section 3 . Hence this procedure is terminating. Moreover, the resulting finite set of finite algebras must generate the quasivariety 
$\mathcal{Q}$ (by Lemma 3.3), contain only $\mathcal{Q}$-subdirectly irreducible algebras, and not contain any algebra that embeds into another member of the set (lines 22 26). Hence by Theorem 3.4, we obtain:

Theorem 3.8. For a finite set $\mathcal{K}$ of finite $\mathcal{L}$-algebras, MinGenSet $(\mathcal{K})$ returns the (unique up to isomorphism) minimal generating set for the quasivariety $\mathbb{Q}(\mathcal{K})$.

We remark that although the algorithm MinGenSeT does not need to calculate the $\mathcal{Q}$ congruence lattice, already calculating the congruence lattice of a finite algebra can take exponential time. Moreover, we make frequent use in our algorithm here of checking embeddings, which is in general an NP-complete problem (see [33, 7]).

\section{Admissibility and Free Algebras}

Let us fix a class of $\mathcal{L}$-algebras $\mathcal{K}$. A homomorphism $\sigma: \mathbf{T m}_{\mathcal{L}} \rightarrow \mathbf{T m}_{\mathcal{L}}$ is called a $\mathcal{K}$-unifier of a set $\Sigma$ of $\mathcal{L}$-equations if $\models_{\mathcal{K}} \sigma(\varphi) \approx \sigma(\psi)$ for every $(\varphi \approx \psi) \in \Sigma$; in this case, $\Sigma$ is said to be $\mathcal{K}$-unifiable. An $\mathcal{L}$-quasiequation $\Sigma \Rightarrow \varphi \approx \psi$ is $\mathcal{K}$-admissible if every $\mathcal{K}$-unifier of $\Sigma$ is a $\mathcal{K}$-unifier of $\varphi \approx \psi$. In this section, we present characterizations of $\mathcal{K}$-unifiability, $\mathcal{K}$ admissibility, and related properties, emphasizing their close relationship to free algebras. The characterizations obtained will then be used in Section 5 to develop algorithms for checking these properties in the context of finitely generated quasivarieties.

Let us first take a closer look at $\mathcal{K}$-unifiability, noting that a finite set $\Sigma$ of $\mathcal{L}$-equations is $\mathcal{K}$-unifiable if and only if the $\mathcal{L}$-negative clause $\Sigma \Rightarrow \emptyset$ is not $\mathcal{K}$-admissible (equivalently, when $\mathcal{K}$ contains a non-trivial algebra, if and only if the $\mathcal{L}$-quasiequation $\Sigma \Rightarrow x \approx y$ with $x, y$ not occurring in $\Sigma$ is not $\mathcal{K}$-admissible). We will see that for checking $\mathcal{K}$-unifiability, the "optimal" solution is to check satisfiability in the smallest finite subalgebra (if such an algebra exists) of $\mathbf{F}_{\mathcal{K}}(\omega)$.

Lemma 4.1. The following are equivalent for any class $\mathcal{K}^{\prime}$ of $\mathcal{L}$-algebras:

(1) $\Sigma$ is $\mathcal{K}$-unifiable iff $\Sigma$ is $\mathcal{K}^{\prime}$-satisfiable.

(2) $\mathbb{V}^{-}\left(\mathcal{K}^{\prime}\right)=\mathbb{V}^{-}\left(\mathbf{F}_{\mathcal{K}}(\omega)\right)$

Proof. Recall that $\mathbb{V}^{-}\left(\mathcal{K}^{\prime}\right)=\mathbb{V}^{-}\left(\mathbf{F}_{\mathcal{K}}(\omega)\right)$ is equivalent to the condition that an $\mathcal{L}$-negative clause $\Sigma \Rightarrow \emptyset$ is $\mathcal{K}^{\prime}$-valid iff it is $\mathbf{F}_{\mathcal{K}}(\omega)$-valid. However, $\Sigma \Rightarrow \emptyset$ is $\mathcal{K}^{\prime}$-valid iff $\Sigma$ is not $\mathcal{K}^{\prime}$ satisfiable and $\Sigma \Rightarrow \emptyset$ is $\mathbf{F}_{\mathcal{K}}(\omega)$-valid iff $\Sigma$ is not $\mathbf{F}_{\mathcal{K}}(\omega)$-satisfiable. For the equivalence of (1) and (2), it suffices therefore to show that $\Sigma$ is $\mathbf{F}_{\mathcal{K}}(\omega)$-satisfiable iff $\Sigma$ is $\mathcal{K}$-unifiable. Suppose first that $h: \mathbf{T m}_{\mathcal{L}} \rightarrow \mathbf{F}_{\mathcal{K}}(\omega)$ satisfies $\Sigma$. Then any homomorphism $\sigma: \mathbf{T m}_{\mathcal{L}} \rightarrow \mathbf{T m}_{\mathcal{L}}$ defined such that $\sigma(x) \in h(x)$ for each variable $x$ is a $\mathcal{K}$-unifier of $\Sigma$. Conversely, if $\sigma$ is a $\mathcal{K}$-unifier of $\Sigma$, then the homomorphism $h: \mathbf{T}_{\mathcal{L}} \rightarrow \mathbf{F}_{\mathcal{K}}(\omega)$ defined by $h(x)=\sigma(x) / \theta_{\mathcal{K}}(\omega)$ for each variable $x$ satisfies $\Sigma$.

Proposition 4.2. Let $\mathcal{K}$ be a class of $\mathcal{L}$-algebras and $\mathbf{C} \in \mathbb{S}\left(\mathbf{F}_{\mathcal{K}}(\omega)\right)$.

(a) $\Sigma$ is $\mathcal{K}$-unifiable iff $\Sigma$ is $\mathbf{C}$-satisfiable.

(b) If $\mathbf{C}$ is a smallest finite subalgebra of $\mathbf{F}_{\mathcal{K}}(\omega)$ and $\mathcal{K}^{\prime}$ is a class of $\mathcal{L}$-algebras such that $\Sigma$ is $\mathcal{K}$-unifiable iff $\Sigma$ is $\mathcal{K}^{\prime}$-satisfiable, then $|C| \leq|B|$ for each $\mathbf{B} \in \mathcal{K}^{\prime}$.

Proof. (a) By assumption, $\mathbf{C} \in \mathbb{V}^{-}\left(\mathbf{F}_{\mathcal{K}}(\omega)\right)$, so $\mathbb{V}^{-}(\mathbf{C}) \subseteq \mathbb{V}^{-}\left(\mathbf{F}_{\mathcal{K}}(\omega)\right)$. But also, since $\mathbf{C} \in$ $\mathbb{S}\left(\mathbf{F}_{\mathcal{K}}(\omega)\right) \subseteq \mathbb{V}\left(\mathbf{F}_{\mathcal{K}}(\omega)\right)=\mathbb{V}(\mathcal{K})$ and $\mathbf{F}_{\mathcal{K}}(\omega)=\mathbf{F}_{\mathbb{V}(\mathcal{K})}(\omega)$ has the universal mapping property for $\mathbb{V}(\mathcal{K})$ over countably infinitely many generators, we obtain a homomorphism $h: \mathbf{F}_{\mathcal{K}}(\omega) \rightarrow$ C defined by $h(x)=c$ for every variable $x$ for some fixed $c \in C$. Hence $h\left[\mathbf{F}_{\mathcal{K}}(\omega)\right]$ is a 
subalgebra of $\mathbf{C}$ and $\mathbf{F}_{\mathcal{K}}(\omega) \in \mathbb{H}^{-1} \mathbb{S}(\mathbf{C}) \subseteq \mathbb{V}^{-}(\mathbf{C})$. So $\mathbb{V}^{-}\left(\mathbf{F}_{\mathcal{K}}(\omega)\right) \subseteq \mathbb{V}^{-}(\mathbf{C})$ and the result follows by Lemma 4.1 .

(b) Let $\mathbf{C}$ be a smallest finite subalgebra of $\mathbf{F}_{\mathcal{K}}(\omega)$ and suppose that $\mathcal{K}^{\prime}$ is a class of $\mathcal{L}$-algebras such that $\Sigma$ is $\mathcal{K}$-unifiable iff $\Sigma$ is $\mathcal{K}^{\prime}$-satisfiable. Then by Lemma 4.1 and part (a), $\mathbb{V}^{-}\left(\mathcal{K}^{\prime}\right)=\mathbb{V}^{-}\left(\mathbf{F}_{\mathcal{K}}(\omega)\right)=\mathbb{V}^{-}(\mathbf{C})$. Hence if $\mathbf{B} \in \mathcal{K}^{\prime} \subseteq \mathbb{V}^{-}\left(\mathcal{K}^{\prime}\right)=\mathbb{V}^{-}\left(\mathbf{F}_{\mathcal{K}}(\omega)\right)=\mathbb{V}^{-}(\mathbf{C})=$ $\mathbb{H}^{-1} \mathbb{S P}_{U}^{*}(\mathbf{C})=\mathbb{H}^{-1}(\mathbf{C})$, then clearly $|C| \leq|B|$.

Example 4.3. The variety DMA of De Morgan algebras is generated as a quasivariety by the 4-element algebra $\mathbf{D}_{4}=\langle\{\perp, a, b, \top\}, \wedge, \vee, \neg, \perp, \top\rangle$ consisting of a distributive bounded lattice with an involutive negation defined as follows:

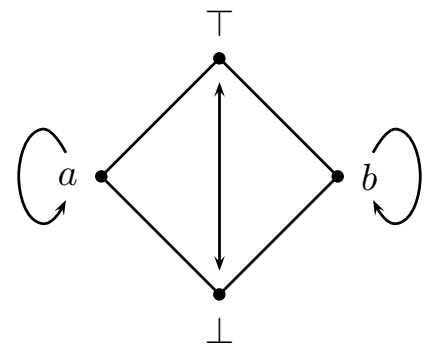

Since there are constants in the language of $\mathbf{D}_{\mathbf{4}}$, the smallest algebra for checking DMAunifiability is the 2-element ground algebra $\mathbf{F}_{\mathbf{D}_{4}}(0)$ : i.e., the 2-element Boolean algebra. That is, checking unifiability amounts to checking classical satisfiability. E.g., $x \wedge \neg x \approx x \vee \neg x$ is not DMA-unifiable, since in the 2-element Boolean algebra, $T \wedge \neg \top \neq \top \vee \neg \top$ and $\perp \wedge \neg \perp \neq \perp \vee \neg \perp$. The case of the "constant-free" variety DML of De Morgan lattices, generated as a quasivariety by $\mathbf{D}_{4}^{\ell}=\langle\{\perp, a, b, \top\}, \wedge, \vee, \neg\rangle$, is not so immediate. However, there is also a smallest 2-element subalgebra of $\mathbf{F}_{\mathbf{D}_{4}^{\ell}}(\omega)$ with elements corresponding to $x \wedge \neg x$ and $x \vee \neg x$. So checking DML-unifiability amounts again to checking classical satisfiability.

Let us turn our attention now to admissible quasiequations, recalling the following useful characterizations of admissibility in terms of free algebras and generated subvarieties.

Theorem 4.4 ([11, Theorem 2]; see also [35, Theorem 1.4.5]). The following are equivalent:

(1) $\Sigma \Rightarrow \varphi \approx \psi$ is $\mathcal{K}$-admissible.

(2) $\Sigma \Rightarrow \varphi \approx \psi$ is $\mathbb{Q}(\mathcal{K})$-admissible.

(3) $\Sigma \models F_{\mathbf{F}_{\mathcal{K}}(\omega)} \varphi \approx \psi$.

(4) $\mathbb{V}(\mathcal{K})=\mathbb{V}\left(\left\{\mathbf{A} \in \mathbb{Q}(\mathcal{K}): \Sigma \models_{\mathbf{A}} \varphi \approx \psi\right\}\right)$.

Example 4.5. The following quasiequations, expressing meet and join semi-distributivity in the language of lattices with $\wedge$ and $\vee$, are satisfied by all free lattices (see [28]), and are therefore admissible in the variety of lattices:

$$
\begin{aligned}
& \{x \wedge y \approx x \wedge z\} \Rightarrow x \wedge y \approx x \wedge(y \vee z) \\
& \{x \vee y \approx x \vee z\} \Rightarrow x \vee y \approx x \vee(y \wedge z) .
\end{aligned}
$$

Similarly, the quasiequations below expressing torsion-freeness in a language of groups with $\cdot,^{-1}$, and e are satisfied by all free groups, and are hence admissible in the variety of groups:

$$
\{\underbrace{x \cdot \ldots \cdot x}_{n} \approx \mathrm{e}\} \Rightarrow x \approx \mathrm{e} \quad(n=2,3, \ldots) .
$$


Given a class $\mathcal{K}$ of $\mathcal{L}$-algebras, we will be interested in determining when the $\mathcal{K}$-admissibility of quasiequations coincides with their $\mathcal{K}^{\prime}$-validity in another class of $\mathcal{L}$-algebras $\mathcal{K}^{\prime}$. By Theorem 4.4, this is the case exactly when $\mathbb{Q}\left(\mathcal{K}^{\prime}\right)=\mathbb{Q}\left(\mathbf{F}_{\mathcal{K}}(\omega)\right)$. The next result provides a further useful characterization of this situation.

Proposition 4.6. The following are equivalent:

(1) $\Sigma \Rightarrow \varphi \approx \psi$ is $\mathcal{K}$-admissible iff $\Sigma \models_{\mathcal{K}^{\prime}} \varphi \approx \psi$.

(2) $\mathbb{Q}\left(\mathcal{K}^{\prime}\right)=\mathbb{Q}\left(\mathbf{F}_{\mathcal{K}}(\omega)\right)$

(3) $\mathcal{K}^{\prime} \subseteq \mathbb{Q}\left(\mathbf{F}_{\mathcal{K}}(\omega)\right)$ and $\mathcal{K} \subseteq \mathbb{V}\left(\mathcal{K}^{\prime}\right)$.

Proof. (1) $\Leftrightarrow(2)$ Follows directly from Theorem 4.4 ,

$(2) \Rightarrow(3)$ Suppose that $\mathbb{Q}\left(\mathcal{K}^{\prime}\right)=\mathbb{Q}\left(\mathbf{F}_{\mathcal{K}}(\omega)\right)$. Then $\mathcal{K}^{\prime} \subseteq \mathbb{Q}\left(\mathbf{F}_{\mathcal{K}}(\omega)\right)$. Moreover, $\mathbb{V}\left(\mathcal{K}^{\prime}\right)=$ $\mathbb{V}\left(\mathbb{Q}\left(\mathcal{K}^{\prime}\right)\right)=\mathbb{V}\left(\mathbb{Q}\left(\mathbf{F}_{\mathcal{K}}(\omega)\right)\right)=\mathbb{V}\left(\mathbf{F}_{\mathcal{K}}(\omega)\right)=\mathbb{V}(\mathcal{K})$, so $\mathcal{K} \subseteq \mathbb{V}\left(\mathcal{K}^{\prime}\right)$.

$(3) \Rightarrow(2)$ Suppose that $\mathcal{K}^{\prime} \subseteq \mathbb{Q}\left(\mathbf{F}_{\mathcal{K}}(\omega)\right)$ and $\mathcal{K} \subseteq \mathbb{V}\left(\mathcal{K}^{\prime}\right)$. Then clearly $\mathbb{Q}\left(\mathcal{K}^{\prime}\right) \subseteq \mathbb{Q}\left(\mathbf{F}_{\mathcal{K}}(\omega)\right)$. But also $\mathbb{V}(\mathcal{K}) \subseteq \mathbb{V}\left(\mathcal{K}^{\prime}\right) \subseteq \mathbb{V}\left(\mathbb{Q}\left(\mathbf{F}_{\mathcal{K}}(\omega)\right)\right)=\mathbb{V}\left(\mathbf{F}_{\mathcal{K}}(\omega)\right)=\mathbb{V}(\mathcal{K})$. That is, $\mathbb{V}(\mathcal{K})=\mathbb{V}\left(\mathcal{K}^{\prime}\right)$. Hence $\mathbf{F}_{\mathcal{K}}(\omega)=\mathbf{F}_{\mathcal{K}^{\prime}}(\omega) \in \mathbb{Q}\left(\mathcal{K}^{\prime}\right)$ and $\mathbb{Q}\left(\mathbf{F}_{\mathcal{K}}(\omega)\right) \subseteq \mathbb{Q}\left(\mathcal{K}^{\prime}\right)$.

For some well-behaved classes of algebras, admissibility and validity coincide. More precisely, a class $\mathcal{K}$ of $\mathcal{L}$-algebras is said to be structurally complete if it satisfies the condition:

$$
\Sigma \Rightarrow \varphi \approx \psi \text { is } \mathcal{K} \text {-admissible } \quad \text { iff } \quad \Sigma \models_{\mathcal{K}} \varphi \approx \psi
$$

The following characterization then follows almost immediately from Theorem 4.4;

Proposition 4.7 ([6, Proposition 2.3]). The following are equivalent:

(1) $\mathcal{K}$ is structurally complete.

(2) $\mathbb{Q}(\mathcal{K})=\mathbb{Q}\left(\mathbf{F}_{\mathcal{K}}(\omega)\right)$.

(3) $\mathcal{K}^{\prime} \subseteq \mathcal{K}$ and $\mathbb{V}\left(\mathcal{K}^{\prime}\right)=\mathbb{V}(\mathcal{K})$ implies $\mathbb{Q}\left(\mathcal{K}^{\prime}\right)=\mathbb{Q}(\mathcal{K})$.

Example 4.8. The variety BA of Boolean algebras, generated as a quasivariety by the 2-element algebra $\mathbf{2}=\langle\{0,1\}, \wedge, \vee, \neg, 0,1\rangle$, is structurally complete. It suffices to observe that $\mathbf{2}$ embeds into $\mathbf{F}_{\mathbf{2}}(\omega)=\mathbf{F}_{\mathrm{BA}}(\omega)$ via a mapping that sends 0 and 1 to their respective equivalence classes, and hence that $\mathrm{BA}=\mathbb{Q}(\mathbf{2})=\mathbb{Q}\left(\mathbf{F}_{\mathrm{BA}}(\omega)\right)$.

Example 4.9. A modular lattice $\mathbf{L}$ may be characterized as a lattice satisfying the equation $(x \wedge y) \vee(y \wedge z) \approx y \wedge((x \wedge y) \vee z)$. Famously, a lattice $\mathbf{L}$ is non-modular if and only if the lattice $\mathbf{N}_{5}$ (below) embeds into $\mathbf{L}$ (see [10, Theorem I.3.5]). But since $\mathbf{N}_{5}$ is nonmodular, also $\mathbf{F}_{\mathbf{N}_{\mathbf{5}}}(\omega)$ (which must satisfy the same equations) is non-modular. So $\mathbf{N}_{\mathbf{5}}$ embeds into $\mathbf{F}_{\mathbf{N}_{5}}(\omega)$, and $\mathbb{Q}\left(\mathbf{N}_{5}\right)$ is structurally complete. Similarly, it is well-known that a lattice $\mathbf{L}$ is distributive if and only if neither $\mathbf{N}_{\mathbf{5}}$ nor $\mathbf{M}_{\mathbf{5}}$ (below) embeds into $\mathbf{L}$ (see [10, Theorem I.3.6]). Since $\mathbf{M}_{\mathbf{5}}$ is non-distributive and modular, also $\mathbf{F}_{\mathbf{M}_{\mathbf{5}}}(\omega)$ is non-distributive and modular. So $\mathbf{M}_{\mathbf{5}}$ embeds into $\mathbf{F}_{\mathbf{M}_{\mathbf{5}}}(\omega)$, and $\mathbb{Q}\left(\mathbf{M}_{\mathbf{5}}\right)$ is structurally complete.
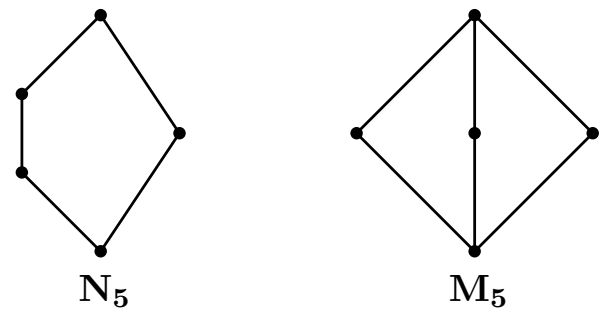
For certain other classes, admissibility and validity coincide for quasiequations with unifiable premises. More precisely, we call a class $\mathcal{K}$ of $\mathcal{L}$-algebras almost structurally complete if it satisfies the condition:

$$
\Sigma \Rightarrow \varphi \approx \psi \text { is } \mathcal{K} \text {-admissible } \quad \text { iff } \quad \Sigma \models_{\mathcal{K}} \varphi \approx \psi \text { or } \Sigma \text { is not } \mathcal{K} \text {-unifiable. }
$$

The next result provides a useful characterization of this situation:

Theorem 4.10. The following are equivalent for any $\mathbf{B} \in \mathbb{S}\left(\mathbf{F}_{\mathcal{K}}(\omega)\right)$ :

(1) $\mathcal{K}$ is almost structurally complete.

(2) $\mathbb{Q}(\{\mathbf{A} \times \mathbf{B}: \mathbf{A} \in \mathcal{K}\})=\mathbb{Q}\left(\mathbf{F}_{\mathcal{K}}(\omega)\right)$.

(3) $\{\mathbf{A} \times \mathbf{B}: \mathbf{A} \in \mathcal{K}\} \subseteq \mathbb{Q}\left(\mathbf{F}_{\mathcal{K}}(\omega)\right)$.

Proof. (1) $\Rightarrow(2)$ Suppose that $\mathcal{K}$ is almost structurally complete. To establish $\mathbb{Q}(\{\mathbf{A} \times \mathbf{B}$ : $\mathbf{A} \in \mathcal{K}\})=\mathbb{Q}\left(\mathbf{F}_{\mathcal{K}}(\omega)\right)$, it suffices to show that a quasiequation $\Sigma \Rightarrow \varphi \approx \psi$ is valid in all algebras $\mathbf{A} \times \mathbf{B}$ for $\mathbf{A} \in \mathcal{K}$ iff it is valid in $\mathbf{F}_{\mathcal{K}}(\omega)$. Suppose first that $\Sigma \models_{\mathbf{F}_{\mathcal{K}}(\omega)} \varphi \approx \psi$. Then by Theorem 4.4, either $\Sigma$ is not $\mathcal{K}$-unifiable or $\Sigma \Rightarrow \varphi \approx \psi$ is $\mathcal{K}$-valid. In the first case, by Proposition 4.2, $\Sigma$ is not $\mathbf{B}$-satisfiable, so $\Sigma \Rightarrow \varphi \approx \psi$ is valid in $\mathbf{A} \times \mathbf{B}$ for all $\mathbf{A} \in \mathcal{K}$. In the second case, $\Sigma \Rightarrow \varphi \approx \psi$ is valid in $\mathbf{A} \times \mathbf{B} \in \mathbb{Q}(\mathcal{K})$ for all $\mathbf{A} \in \mathcal{K}$. Conversely, if $\Sigma \Rightarrow \varphi \approx \psi$ is valid in $\mathbf{A} \times \mathbf{B}$ for each $\mathbf{A} \in \mathcal{K}$, then either $\Sigma$ is not $\mathbf{B}$-satisfiable or $\Sigma \Rightarrow \varphi \approx \psi$ is valid in each $\mathbf{A}$ in $\mathcal{K}$. In the first case, by Proposition 4.2, $\Sigma$ is not $\mathcal{K}$-unifiable, so $\Sigma \Rightarrow \varphi \approx \psi$ is valid in $\mathbf{F}_{\mathcal{K}}(\omega)$. In the second case, $\Sigma \Rightarrow \varphi \approx \psi$ is valid in $\mathbb{Q}(\mathcal{K})$ and hence valid in $\mathbf{F}_{\mathcal{K}}(\omega)$.

$(2) \Rightarrow(1)$ Suppose that $\mathbb{Q}\left(\mathbf{F}_{\mathcal{K}}(\omega)\right)=\mathbb{Q}(\{\mathbf{A} \times \mathbf{B}: \mathbf{A} \in \mathcal{K}\})$. Then whenever $\Sigma \Rightarrow \varphi \approx \psi$ is $\mathcal{K}$-admissible, it is $\mathbf{F}_{\mathcal{K}}(\omega)$-valid and hence also valid in $\mathbf{A} \times \mathbf{B}$ for all $\mathbf{A} \in \mathcal{K}$. Moreover, if $\Sigma$ is $\mathcal{K}$-unifiable, then, by Proposition 4.2, it is $\mathbf{B}$-satisfiable. I.e., there exists a homomorphism $h: \mathbf{T}_{\mathcal{L}} \rightarrow \mathbf{B}$ with $\Sigma \subseteq \operatorname{ker} h$. For any $\mathbf{A} \in \mathcal{K}$ and homomorphism $k: \mathbf{T m}_{\mathcal{L}} \rightarrow \mathbf{A}$ with $\Sigma \subseteq$ ker $k$, define $e_{\mathbf{A}}: \mathbf{T m}_{\mathcal{L}} \rightarrow \mathbf{A} \times \mathbf{B}$ by $e_{\mathbf{A}}(u)=(k(u), h(u))$. Then, since $\Sigma \Rightarrow \varphi \approx \psi$ is valid in $\mathbf{A} \times \mathbf{B}$ for all $\mathbf{A} \in \mathcal{K}, \Sigma \subseteq \operatorname{ker} e$, so $e(\varphi)=e(\psi)$ and $k(\varphi)=k(\psi)$. I.e., $\Sigma \models_{\mathbf{A}} \varphi \approx \psi$. So we have shown that $\Sigma \models \mathcal{K} \varphi \approx \psi$.

$(2) \Rightarrow(3)$ Immediate.

(3) $\Rightarrow(2)$ Suppose that $\{\mathbf{A} \times \mathbf{B}: \mathbf{A} \in \mathcal{K}\} \subseteq \mathbb{Q}\left(\mathbf{F}_{\mathcal{K}}(\omega)\right)$. Then also, since $\mathbf{A} \in \mathbb{H}(\mathbf{A} \times \mathbf{B})$ for each $\mathbf{A} \in \mathcal{K}$, we obtain $\mathcal{K} \subseteq \mathbb{V}(\{\mathbf{A} \times \mathbf{B}: \mathbf{A} \in \mathcal{K}\})$. Hence by Proposition 4.6, $\mathbb{Q}(\{\mathbf{A} \times \mathbf{B}$ : $\mathbf{A} \in \mathcal{K}\})=\mathbb{Q}\left(\mathbf{F}_{\mathcal{K}}(\omega)\right)$

Example 4.11. Consider the 2-element and 3-element Wajsberg algebras $\mathbf{L}_{\mathbf{2}}=\langle\{0,1\}, \rightarrow$ $, \neg\rangle$ and $\mathbf{L}_{\mathbf{3}}=\left\langle\left\{0, \frac{1}{2}, 1\right\}, \rightarrow, \neg\right\rangle$ where

$$
x \rightarrow y=\min (1,1-x+y) \quad \text { and } \neg x=1-x .
$$

The algebra $\mathbf{L}_{\mathbf{3}} \times \mathbf{L}_{\mathbf{2}}$ embeds into $\mathbf{F}_{\mathbf{L}_{\mathbf{3}}}(\omega)$, as illustrated in the diagram below by the terms associated to elements, and has $\mathbf{L}_{\mathbf{3}}$ as a homomorphic image, as indicated by the arrows. Hence $\mathbf{L}_{\mathbf{3}}$ is almost structurally complete. Note that it is not structurally complete since, for example, $\{x \approx \neg x\} \Rightarrow \emptyset$ is $\mathbf{L}_{\mathbf{3}}$-admissible, but not $\mathbf{L}_{\mathbf{3}}$-valid. On the other hand, its implicational reduct $\mathbf{L}_{\mathbf{3}}=\left\langle\left\{0, \frac{1}{2}, 1\right\}, \rightarrow\right\rangle$ is structurally complete, since it embeds into the free algebra $\mathbf{F}_{\mathbf{L}_{\mathbf{3}}}(2)$. 


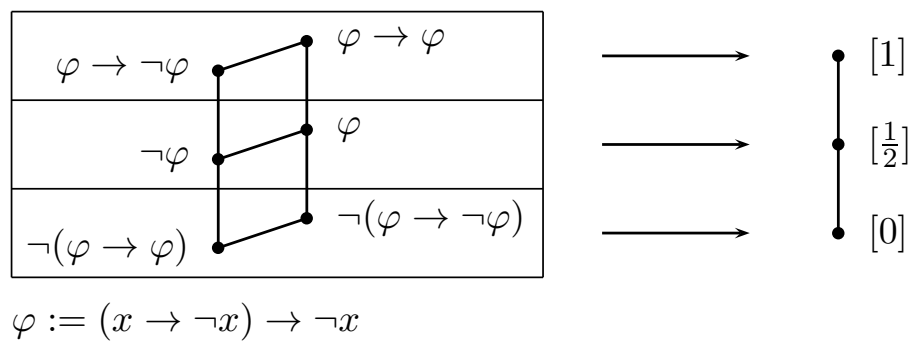

\section{Algorithms And Examples}

In this section, we present algorithms for checking admissibility of quasiequations and related properties in finitely generated quasivarieties. We also present results on admissibility (some new, some well-known) for a range of examples taken from the universal algebra and non-classical logic literature. These results, obtained using a Delphi implementation of the algorithms described here, are collected at the end of this section in Table 1.

We begin by recalling Birkhoff's result that the finitely generated free algebras for a finite set $\mathcal{K}$ of finite algebras are themselves finite; more precisely:

Lemma 5.1 ([8]). For any set of finite algebras $\mathcal{K}=\left\{\mathbf{A}_{1}, \ldots, \mathbf{A}_{m}\right\}$ and $n \in \mathbb{N}$ :

$$
\left|F_{\mathcal{K}}(n)\right| \leq \prod_{i=1}^{m}\left|A_{i}\right|^{\left|A_{i}\right|^{n}} .
$$

Hence for the relatively easy task of checking $\mathcal{K}$-unifiability, it suffices to find (e.g., through exhaustive search) the (unique up to isomorphism) smallest subalgebra $\mathbf{C}$ of the finite free algebra $\mathbf{F}_{\mathcal{K}}(1)$, noting that this is $\mathbf{F}_{\mathcal{K}}(0)$ if the language contains constants. It then follows by Proposition 4.2 that a set of equations $\Sigma$ is $\mathcal{K}$-unifiable if and only if $\Sigma$ is $\mathbf{C}$-valid, and indeed that there is no smaller algebra with this property.

For checking $\mathcal{K}$-admissibility, we make use of a known result for finitely generated quasivarieties (see [35, Lemma 4.1.10]), obtained here as a corollary of Proposition 4.6.

Corollary 5.2. Given a finite set $\mathcal{K}$ of finite $\mathcal{L}$-algebras with $n=\max \{|A|: \mathbf{A} \in \mathcal{K}\}$ :

(a) $\mathbb{Q}\left(\mathbf{F}_{\mathcal{K}}(\omega)\right)=\mathbb{Q}\left(\mathbf{F}_{\mathcal{K}}(n)\right)$.

(b) $\Sigma \Rightarrow \varphi \approx \psi$ is $\mathcal{K}$-admissible iff $\Sigma \models_{\mathbf{F}_{\mathcal{K}}(n)} \varphi \approx \psi$.

Proof. Observe first that each $\mathbf{A} \in \mathcal{K}$ is a homomorphic image of $\mathbf{F}_{\mathcal{K}}(n)$. That is, define any surjective map from the $n$ generators of $\mathbf{F}_{\mathcal{K}}(n)$ to $A$; this extends to a homomorphism from $\mathbf{F}_{\mathcal{K}}(n)$ onto $\mathbf{A}$ since $\mathbf{F}_{\mathcal{K}}(n)$ has the universal mapping property for $\mathcal{K}$ over $n$ generators. So $\mathcal{K} \subseteq \mathbb{V}\left(\mathbf{F}_{\mathcal{K}}(n)\right)$ and, since also $\mathbf{F}_{\mathcal{K}}(n) \in \mathbb{Q}\left(\mathbf{F}_{\mathcal{K}}(\omega)\right)$, (a) and (b) follow by Proposition 4.6.

Hence checking $\mathcal{K}$-admissibility of quasiequations is decidable. However, even when $\mathcal{K}$ consists of a small number of small algebras, free algebras on a small number of generators can be quite large. For example, the free algebra $\mathbf{F}_{\mathbf{D}_{4}}(2)$ (see Example 4.3) has 168 elements. We therefore seek smaller algebras or finite sets of smaller algebras that also generate $\mathbb{Q}\left(\mathbf{F}_{\mathcal{K}}(\omega)\right)$ as a quasivariety. In fact, since $\mathbb{Q}\left(\mathbf{F}_{\mathcal{K}}(\omega)\right)$ is finitely generated, we may apply the multiset ordering defined in Section 3, and seek a minimal generating set of finite algebras for this quasivariety that is unique up to isomorphism. One strategy would therefore be to apply the algorithm MinGenSeT directly to $\mathbf{F}_{\mathcal{K}}(n)$. However, this method is not feasible 


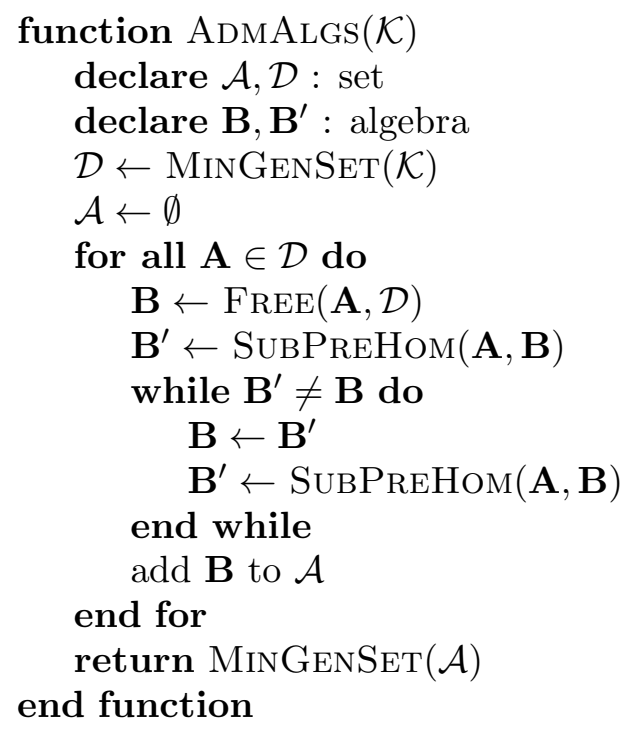

Figure 2: For a finite set $\mathcal{K}$ of finite algebras, return the minimal generating set of $\mathbb{Q}\left(\mathbf{F}_{\mathcal{K}}(\omega)\right)$.

for large free algebras, since it involves the computationally labour-intensive task of building the congruence lattice of $\mathbf{F}_{\mathcal{K}}(n)$. Instead, we make auxiliary use of the following immediate corollary of Proposition 4.6:

Corollary 5.3. Given a class $\mathcal{K}$ of $\mathcal{L}$-algebras and $\mathcal{K}^{\prime} \subseteq \mathbb{S}\left(\mathbf{F}_{\mathcal{K}}(\omega)\right)$ such that $\mathcal{K} \subseteq \mathbb{H}\left(\mathcal{K}^{\prime}\right)$ :

(a) $\mathbb{Q}\left(\mathcal{K}^{\prime}\right)=\mathbb{Q}\left(\mathbf{F}_{\mathcal{K}}(\omega)\right)$.

(b) $\Sigma \Rightarrow \varphi \approx \psi$ is $\mathcal{K}$-admissible iff $\Sigma \models \mathcal{K}^{\prime} \varphi \approx \psi$.

We describe an algorithm AdmAlgs (see Figure 2) which takes as input a finite set $\mathcal{K}$ of finite $\mathcal{L}$-algebras and outputs the (unique up to isomorphism) minimal generating set for $\mathbb{Q}\left(\mathbf{F}_{\mathcal{K}}(\omega)\right.$ ). First, the procedure MinGenSet is applied to $\mathcal{K}$ (which typically would be a small set of small algebras) to obtain the set of $\mathcal{L}$-algebras $\mathcal{D}$. Then for each $\mathbf{A} \in \mathcal{D}$, a procedure $\operatorname{FrEE}(\mathbf{A}, \mathcal{D})$ is invoked to produce the smallest free algebra $\mathbf{F}_{\mathcal{D}}(m)$ having $\mathbf{A}$ as a homomorphic image. (The procedure begins by checking the smallest free algebra $\mathbf{F}_{\mathcal{D}}(0)$ or $\mathbf{F}_{\mathcal{D}}(1)$, then increases the number of generators one at a time.) The algorithm then searches for progressively smaller subalgebras of $\mathbf{F}_{\mathcal{D}}(m)$ which have $\mathbf{A}$ as a homomorphic image. More precisely, the procedure $\operatorname{SubPreHom}(\mathbf{A}, \mathbf{B})$ searches for a proper subalgebra of $\mathbf{B}$ that is a homomorphic image of $\mathbf{A}$, returning $\mathbf{B}$ if no such algebra exists (line 9). This process terminates with a (hopefully reasonably small) algebra which is added to a set $\mathcal{A}$. Finally, the procedure MinGenSet is applied to $\mathcal{A}$.

Theorem 5.4. For a finite set $\mathcal{K}$ of finite $\mathcal{L}$-algebras, $\operatorname{AdmAlgs}(\mathcal{K})$ returns the (unique up to isomorphism) minimal generating set for the quasivariety $\mathbb{Q}\left(\mathbf{F}_{\mathcal{K}}(\omega)\right)$.

Example 5.5. Note that given even just one algebra $\mathbf{A}$, it might not be the case that the smallest subalgebra of $\mathbf{F}_{\mathbf{A}}(\omega)$ having $\mathbf{A}$ as a homomorphic image is the smallest algebra that generates $\mathbb{Q}\left(\mathbf{F}_{\mathbf{A}}(\omega)\right)$. Consider the 4-element algebra $\mathbf{P}=\langle\{a, b, c, d\}, \star\rangle$ where the unary function $\star$ and the free algebras $\mathbf{F}_{\mathbf{P}}(n)$ are described by the following diagrams: 


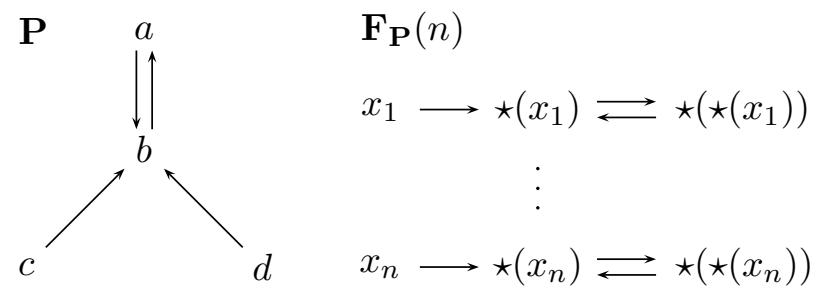

The smallest subalgebra of $\mathbf{F}_{\mathbf{P}}(\omega)$ with $\mathbf{P}$ as a homomorphic image is the 6-element free algebra $\mathbf{F}_{\mathbf{P}}(2)$. However, MinGenSet $(\{\mathbf{P}\})$, performed at the beginning of the algorithm AdmAlgs, discovers that $\mathbf{P}$ is a $\mathbb{Q}(\mathbf{P})$-subdirect product of (two copies of) the 3 -element free algebra $\mathbf{F}_{\mathbf{P}}(1)$. Hence $\mathbb{Q}(\mathbf{P})=\mathbb{Q}\left(\mathbf{F}_{\mathbf{P}}(1)\right)$ and this quasivariety is structurally complete.

In the previous example, the fact that the algebra $\mathbf{P}$ is structurally complete is discovered by the algorithm ADMAlgs. However, structural completeness can also be checked more directly using the following result:

Proposition 5.6. The following are equivalent for any finite set $\mathcal{K}$ of finite $\mathcal{L}$-algebras:

(1) $\mathcal{K}$ is structurally complete.

(2) $\operatorname{MinGenSet}(\mathcal{K}) \subseteq \mathbb{I S}\left(\mathbf{F}_{\mathcal{K}}(n)\right)$ where $n=\max \{|C|: \mathbf{C} \in \mathcal{K}\}$.

Proof. (1) $\Rightarrow$ (2) If $\mathcal{K}$ is structurally complete, then, by Proposition 4.7 and Corollary 5.2 , $\mathbb{Q}(\mathcal{K})=\mathbb{Q}\left(\mathbf{F}_{\mathcal{K}}(\omega)\right)=\mathbb{Q}\left(\mathbf{F}_{\mathcal{K}}(n)\right)$ where $n=\max \{|C|: \mathbf{C} \in \mathcal{K}\}$. So MinGenSet $(\mathcal{K}) \subseteq$ $\mathbb{Q}\left(\mathbf{F}_{\mathcal{K}}(n)\right)$. But each $\mathbf{A} \in \operatorname{Min} \operatorname{GenSet}(\mathcal{K})$ is $\mathbb{Q}\left(\mathbf{F}_{\mathcal{K}}(n)\right)$-subdirectly irreducible, so by Lemma 3.2, A embeds into $\mathbf{F}_{\mathcal{K}}(n)$. I.e., $\operatorname{MinGenSet}(\mathcal{K}) \subseteq \mathbb{I S}\left(\mathbf{F}_{\mathcal{K}}(n)\right)$.

$(2) \Rightarrow(1)$ If each $\mathbf{A} \in \operatorname{MinGenSet}(\mathcal{K})$ embeds into $\mathbf{F}_{\mathcal{K}}(n)$, then $\mathbb{Q}\left(\mathbf{F}_{\mathcal{K}}(n)\right) \subseteq \mathbb{Q}(\mathcal{K})=$ $\mathbb{Q}(\operatorname{MinGenSet}(\mathcal{K})) \subseteq \mathbb{Q}\left(\mathbf{F}_{\mathcal{K}}(n)\right)$. So $\mathcal{K}$ is structurally complete by Proposition 4.7.

Example 5.7. Proposition 5.6 has been used to confirm known structural completeness results for the 3 -element positive Gödel algebra $\mathbf{G}_{\mathbf{3}}^{+}=\left\langle\left\{0, \frac{1}{2}, 1\right\}\right.$, min, max, $\left.\rightarrow_{\mathrm{G}}\right\rangle$ where $x \rightarrow_{\mathrm{G}}$ $y$ is $y$ if $x>y$, otherwise 1 , and the Stone algebra $\mathbf{B}_{\mathbf{1}}=\left\langle\left\{0, \frac{1}{2}, 1\right\}\right.$, min, max, $\left.\neg \mathrm{G}\right\rangle$ where $\neg_{\mathrm{G}} x=x \rightarrow_{\mathrm{G}}$ 0. Moreover, a new structural completeness result has been established for the pseudocomplemented distributive lattice $\mathbf{B}_{\mathbf{2}}$, obtained by adding a top element to the 4-element Boolean lattice, and calculating the pseudocomplement.

Similarly, we can check almost structural completeness using the following result:

Proposition 5.8. The following are equivalent for any finite set $\mathcal{K}$ of finite $\mathcal{L}$-algebras and $\mathbf{B} \in \mathbb{S}\left(\mathbf{F}_{\mathcal{K}}(\omega)\right):$

(1) $\mathcal{K}$ is almost structurally complete.

(2) $\operatorname{MinGenSet}(\{\mathbf{A} \times \mathbf{B}: \mathbf{A} \in \mathcal{K}\}) \subseteq \mathbb{I S}\left(\mathbf{F}_{\mathcal{K}}(n)\right)$ where $n=\max \{|C|: \mathbf{C} \in \mathcal{K}\}$.

Proof. (1) $\Rightarrow(2)$ If $\mathcal{K}$ is almost structurally complete, then by Theorem 4.10 and Corollary 5.2, $\mathbb{Q}(\{\mathbf{A} \times \mathbf{B}: \mathbf{A} \in \mathcal{K}\})=\mathbb{Q}\left(\mathbf{F}_{\mathcal{K}}(\omega)\right)=\mathbb{Q}\left(\mathbf{F}_{\mathcal{K}}(n)\right)$ where $n=\max \{|C|: \mathbf{C} \in \mathcal{K}\}$. In particular, MinGenSet $(\{\mathbf{A} \times \mathbf{B}: \mathbf{A} \in \mathcal{K}\}) \subseteq \mathbb{Q}\left(\mathbf{F}_{\mathcal{K}}(n)\right)$. But each $\mathbf{C} \in \operatorname{Min} \operatorname{GenSet}(\{\mathbf{A} \times$ $\mathbf{B}: \mathbf{A} \in \mathcal{K}\})$ is $\mathbb{Q}\left(\mathbf{F}_{\mathcal{K}}(n)\right)$-subdirectly irreducible, so by Lemma 3.2, $\mathbf{C}$ embeds into $\mathbf{F}_{\mathcal{K}}(n)$. I.e., $\operatorname{MinGenSet}(\{\mathbf{A} \times \mathbf{B}: \mathbf{A} \in \mathcal{K}\}) \subseteq \mathbb{I S}\left(\mathbf{F}_{\mathcal{K}}(n)\right)$.

$(2) \Rightarrow(1)$ If MinGenSet $(\{\mathbf{A} \times \mathbf{B}: \mathbf{A} \in \mathcal{K}\}) \subseteq \mathbb{I} S\left(\mathbf{F}_{\mathcal{K}}(n)\right)$, then $\{\mathbf{A} \times \mathbf{B}: \mathbf{A} \in \mathcal{K}\} \subseteq$ $\mathbb{Q}\left(\mathbf{F}_{\mathcal{K}}(n)\right)=\mathbb{Q}\left(\mathbf{F}_{\mathcal{K}}(\omega)\right)$. So by Theorem 4.10, $\mathcal{K}$ is almost structurally complete. 
Example 5.9. The Sobociński algebra $\mathbf{S}_{\mathbf{3}}=\langle\{-1,0,1\}, \rightarrow, \neg\rangle$ with operations

\begin{tabular}{r|rrr}
$\rightarrow$ & -1 & 0 & 1 \\
\hline-1 & 1 & 1 & 1 \\
0 & -1 & 0 & 1 \\
1 & -1 & -1 & 1
\end{tabular}

\begin{tabular}{r|r}
$\neg$ & \\
\hline-1 & 1 \\
0 & 0 \\
1 & -1
\end{tabular}

is a weak characteristic matrix for the multiplicative fragment $\mathrm{RM}_{\mathrm{m}}$ of the substructural logic R-mingle: $\varphi$ is a theorem of $\mathrm{RM}_{\mathrm{m}}$ if and only if $\models_{\mathrm{S}_{3}} \varphi \approx \varphi \rightarrow \varphi$. Using Proposition 5.8, we establish that $\mathbf{S}_{\mathbf{3}}$ is almost structurally complete and that $\mathbb{Q}\left(\mathbf{F}_{\mathbf{S}_{\mathbf{3}}}(\omega)\right)=\mathbb{Q}\left(\mathbf{S}_{\mathbf{3}} \times \mathbf{S}_{\mathbf{2}}\right)$ where $\mathbf{S}_{\mathbf{2}}=\langle\{-1,1\}, \rightarrow, \neg\rangle$. Note, moreover, that the implicational reduct $\mathbf{S}^{\rightarrow}=\langle\{-1,0,1\}, \rightarrow\rangle$ of $\mathbf{S}_{\mathbf{3}}$ is structurally complete, which can be confirmed directly using Proposition 5.6 or discovered automatically by ADMALgs.

Example 5.10. Consider now the algebra $\mathbf{Z}_{\mathbf{4}}=\langle\{-2,-1,1,2\}, \rightarrow, \neg, 1\rangle$ (the reduct of a 4-element algebra for R-mingle) with a constant 1 and operations

\begin{tabular}{r|rrrr}
$\rightarrow$ & -2 & -1 & 1 & 2 \\
\hline-2 & 2 & 2 & 2 & 2 \\
-1 & -2 & 1 & 1 & 2 \\
1 & -2 & -1 & 1 & 2 \\
2 & -2 & -2 & -2 & 2
\end{tabular}

\begin{tabular}{r|r}
$\neg$ & \\
\hline-2 & 2 \\
-1 & 1 \\
1 & -1 \\
2 & -2
\end{tabular}

ADMALGs produces a 6 -element algebra (the product of a 2-element algebra and a 3 -element algebra). Remarkably, if we drop $\neg$ to obtain $\mathbf{Z}_{4}^{+}=\langle\{-2,-1,1,2\}, \rightarrow, 1\rangle$, AdmAlgs produces a 4-element algebra that is not isomorphic to $\mathbf{Z}_{\mathbf{4}}^{+}$.

Example 5.11. Recall from Example 4.3 that the algebras $\mathbf{D}_{\mathbf{4}}^{\ell}$ and $\mathbf{D}_{\mathbf{4}}$ generate the varieties of De Morgan latices and De Morgan algebras, respectively, as quasivarieties. Both $\mathbf{D}_{\mathbf{4}}^{\ell}$ and $\mathbf{D}_{4}$ are homomorphic images of the corresponding free algebras on two generators (with 166 and 168 elements, respectively) but not on one generator. In the De Morgan lattice case, AdmAlgs finds a smallest suitable subalgebra isomorphic to $\mathbf{D}_{\mathbf{4}}^{\ell} \times \mathbf{2}$, while for De Morgan algebras, the smallest suitable algebra is isomorphic to $\mathbf{D}_{\mathbf{4}} \times \mathbf{2}$ with additional top and bottom elements. These results were established "by hand" in [31; our procedure here confirms them automatically. Similar results were also obtained in [31] for Kleene lattices and Kleene algebras (subvarieties of De Morgan lattices and De Morgan algebras, respectively) which are generated as quasivarieties by the 3 -element chains $\mathbf{C}_{\mathbf{3}}^{\ell}=\langle\{\top, e, \perp\}, \wedge, \vee, \neg\rangle$ and $\mathbf{C}_{3}=\langle\{\top, e, \perp\}, \wedge, \vee, \neg, \perp, \top\rangle$ where $\neg$ swaps $\perp$ and $\top$ and leaves $e$ fixed. In both cases, the smallest algebra for $\mathbf{C}_{\mathbf{3}}$ and $\mathbf{C}_{\mathbf{3}}^{\ell}$, found automatically by ADMALGS is a 4-element chain.

Example 5.12. Consider the 2-element and 3-element chains, $\mathbf{C}_{\mathbf{2}}^{\mathbf{e}}=\langle\{\perp, \top\}, \wedge, \vee, \neg, \top\rangle$ and $\mathbf{C}_{\mathbf{3}}^{\mathbf{e}}=\langle\{\perp, e, \top\}, \wedge, \vee, \neg, e\rangle$ where $\neg$ swaps $\perp$ and $\top$ and leaves $e$ fixed. Individually, these algebras are structurally complete. However, applying AdmAlgs to $\mathcal{K}=\left\{\mathbf{C}_{\mathbf{2}}^{\mathbf{e}}, \mathbf{C}_{\mathbf{3}}^{\mathbf{e}}\right\}$, we find that $\mathcal{K}$ is not structurally complete: both $\mathbf{C}_{\mathbf{2}}^{\mathbf{e}}$ and $\mathbf{C}_{\mathbf{3}}^{\mathbf{e}}$ are homomorphic images of the 16-element free algebra $\mathbf{F}_{\mathcal{K}}(1)$, and the minimal generating set for $\mathbb{Q}\left(\mathbf{F}_{\mathcal{K}}(\omega)\right)$ consists of a single 4-element algebra.

We remark finally that except for Example 5.12, all the case studies considered in this section (see Table 1) involve quasivarieties generated by a single algebra with five elements or fewer, and produce minimal generating sets for checking admissibility also containing just one algebra. Indeed in many of the cases considered, the quasivariety is shown to be either structurally complete or almost structurally complete. The main obstacle at present 


\begin{tabular}{|c|c|c|c|c|c|}
\hline $\mathbf{A}$ & $|A|$ & Quasivariety $\mathbb{Q}(\mathbf{A})$ & Free alg & gebra & $|\operatorname{ADmAlgs}(\mathbf{A})|$ \\
\hline 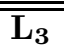 & 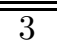 & "algebras for $\mathrm{Ł}_{3}$ (Ex. 4.11) & $\overline{\mid \overline{\left|F_{\mathbf{A}}(1)\right|}}$ & $=12$ & $\overline{66}$ \\
\hline $\overrightarrow{\mathbf{L}_{3}}$ & 3 & algebras for $\mathrm{E}_{3} \rightarrow$ (Ex. 4.11) & $\left|F_{\mathbf{A}}(2)\right|$ & $=40$ & 3 \\
\hline $\mathbf{B}_{1}$ & 3 & Stone algebras (Ex. 5.7) & $\left|F_{\mathbf{A}}(1)\right|$ & $=6$ & 3 \\
\hline $\mathrm{C}_{3}$ & 3 & Kleene algebras (Ex. 5.11) & $\left|F_{\mathbf{A}}(1)\right|$ & $=6$ & 4 \\
\hline $\mathbf{C}_{3}^{\ell}$ & 3 & Kleene lattices (Ex. 5.11) & $\left|F_{\mathbf{A}}(2)\right|$ & $=82$ & 4 \\
\hline $\mathrm{S}_{3}$ & 3 & algebras for $\mathrm{RM} \rightarrow \neg(\mathrm{Ex}$. [5.9) & $\left|F_{\mathbf{A}}(2)\right|$ & $=264$ & 6 \\
\hline $\mathrm{S}_{3}$ & 3 & algebras for $\mathrm{RM}^{\rightarrow}($ Ex. [5.9) & $\left|F_{\mathbf{A}}(2)\right|$ & $=60$ & 3 \\
\hline $\mathrm{G}_{3}^{+}$ & 3 & algebras for $\mathrm{G}_{3}^{+}$(Ex. [5.7) & $\left|F_{\mathbf{A}}(2)\right|$ & $=18$ & 3 \\
\hline$\stackrel{s}{\mathrm{D}_{4}^{\ell}}$ & 4 & De Morgan lattices (Exs 4.315.11) & $\left|F_{\mathbf{A}}(2)\right|$ & $=166$ & 8 \\
\hline$\frac{x_{4}}{D_{4}}$ & 4 & De Morgan algebras (Exs. 4.315.11) & $\left|F_{\mathbf{A}}(2)\right|$ & $=168$ & 10 \\
\hline $\mathbf{P}$ & 4 & $\mathbb{Q}(\mathbf{P})($ Ex. 5.5$)$ & $\left|F_{\mathbf{A}}(2)\right|$ & $=6$ & 3 \\
\hline$\overline{Z_{4}}$ & 4 & algebras for $\mathrm{RM}^{\rightarrow \neg \mathrm{e}}(\mathrm{Ex}$. [5.10) & $\left|F_{\mathbf{A}}(1)\right|$ & $=18$ & 6 \\
\hline$\overline{Z_{4}^{+}}$ & 4 & algebras for $\mathrm{RM}^{\rightarrow \mathrm{e}}$ (Ex. 5.10) & $\left|F_{\mathbf{A}}(2)\right|$ & $=453$ & 4 \\
\hline $\begin{array}{l}\mathbf{4} \\
\mathbf{B}_{2}\end{array}$ & 5 & $\overline{\mathbb{Q}}\left(\mathbf{B}_{2}\right)(\mathrm{Ex} \cdot[5.7)$ & $\left|F_{\mathbf{A}}(1)\right|$ & $=7$ & 5 \\
\hline $\mathrm{M}_{5}$ & 5 & lattices in $\mathbb{Q}\left(\mathbf{M}_{\mathbf{5}}\right)($ Ex. 4.9$)$ & $\left|F_{\mathbf{A}}(3)\right|$ & $=28$ & 5 \\
\hline $\mathbf{N}_{5}$ & 5 & lattices in $\mathbb{Q}\left(\mathbf{N}_{\mathbf{5}}\right)($ Ex. 4.9$)$ & $\left|F_{\mathbf{A}}(3)\right|$ & $=99$ & 5 \\
\hline
\end{tabular}

Table 1: Algebras for checking admissibility

to extending our experimental results to (larger sets of) larger algebras is the size of the resulting free algebras (in the worst case, size $k^{k^{n}}$ for $n$ generators and an algebra of size $k$ ), both for computing these algebras and then for generating suitable subalgebras. Nevertheless, we hope in future work to obtain procedures capable of treating larger examples by using heuristics to constrain the search for appropriate generating algebras.

\section{Finite-VAlued LOGics}

The preceding characterizations of admissibility can be adapted relatively straightforwardly to finite-valued logics. However, in this setting, the designated values of the logic - the "true truth values" - must also be considered, and dealt with appropriately. Here we describe a method that given a finite-valued logic L, provides another (hopefully small) finite-valued logic $L^{\prime}$ such that validity in $L^{\prime}$ corresponds to admissibility in $L$. The more general case, where a smallest finite set of logics is sought such that validity in all members of the set corresponds to admissibility in a logic (or logics), is left as an exercise for the interested reader.

Recall that a finite-valued logic $\mathrm{L}=(\mathbf{A}, D)$ for a language $\mathcal{L}$ consists of a finite $\mathcal{L}$ algebra $\mathbf{A}$ and a set of designated values $D \subseteq A$. Given $\Gamma \cup\{\varphi\} \subseteq \operatorname{Tm}_{\mathcal{L}}$, we let $\Gamma \vdash_{\mathrm{L}} \varphi$ denote that for all homomorphisms $h: \mathbf{T m}_{\mathcal{L}} \rightarrow \mathbf{A}$, whenever $h[\Gamma] \subseteq D$, also $h(\varphi) \in D$. A term $\varphi$ is L-valid if $\vdash_{\mathrm{L}} \varphi$. We call a logic $\mathrm{L}_{1}=\left(\mathbf{A}_{1}, D_{1}\right)$ for a language $\mathcal{L}$ a sublogic of a logic $\mathrm{L}_{2}=\left(\mathbf{A}_{2}, D_{2}\right)$ if $\mathbf{A}_{1}$ is a subalgebra of $\mathbf{A}_{2}$ and also $D_{1}=D_{2} \cap A_{1}$.

Consider now a finite-valued logic $\mathrm{L}=(\mathbf{A}, D)$ for a language $\mathcal{L}$ and a finite set of terms $\Gamma \cup\{\varphi\} \subseteq \operatorname{Tm}_{\mathcal{L}}$. We say that $\Gamma$ is L-unifiable if there exists a homomorphism $\sigma: \mathbf{T}_{\mathcal{L}} \rightarrow \mathbf{T}_{\mathcal{L}}$ such that $\vdash_{\mathrm{L}} \sigma(\psi)$ for all $\psi \in \Gamma$ and call $\sigma$ in this case an L-unifier of $\Gamma$. A rule $\Gamma / \varphi$ is said to be L-admissible if every L-unifier of $\Gamma$ is an L-unifier of $\varphi$. Now if we define the finite-valued logic $\mathrm{L}^{*}=\left(\mathbf{F}_{\mathbf{A}}(|A|), D^{*}\right)$ where $D^{*}=\left\{[\varphi] \in \mathbf{F}_{\mathbf{A}}(|A|): \vdash_{\mathrm{L}} \varphi\right\}$, then we easily obtain the following analogue of Theorem 4.4 . 
Lemma 6.1. Let $\mathrm{L}=(\mathbf{A}, D)$ be a finite-valued logic for a language $\mathcal{L}$. Then $\Gamma / \varphi$ is L-admissible iff $\Gamma \vdash_{\mathrm{L}^{*}} \varphi$.

The next result may then be understood as an analogue of Proposition 4.6.

Proposition 6.2. Let $\mathrm{L}=\left(\mathbf{A}, D_{A}\right)$ be a finite-valued logic for a language $\mathcal{L}$, and let $\mathrm{L}^{\prime}=$ $\left(\mathbf{B}, D_{B}\right)$ be a sublogic of $\mathrm{L}^{*}$ such that there exists a surjective homomorphism $h: \mathbf{B} \rightarrow \mathbf{A}$ satisfying $h\left[D_{B}\right] \subseteq D_{A}$. Then $\Gamma / \varphi$ is L-admissible iff $\Gamma \vdash_{\mathrm{L}^{\prime}} \varphi$.

Proof. If $\Gamma / \varphi$ is L-admissible, then by Lemma 6.1, $\Gamma \vdash_{\mathrm{L}^{*}} \varphi$. Since $\mathrm{L}^{\prime}$ is a sublogic of $\mathrm{L}^{*}$, also $\Gamma \vdash_{\mathrm{L}^{\prime}} \varphi$. Conversely, suppose that $\Gamma \vdash_{\mathrm{L}^{\prime}} \varphi$ and that $\sigma$ is an L-unifier of $\Gamma$. Notice that if $\vdash_{\mathrm{L}} \psi$, then $\vdash_{\mathrm{L}^{*}} \psi$ and $\vdash_{\mathrm{L}^{\prime}} \psi$. So $\sigma$ is also an $\mathrm{L}^{*}$-unifier and $\mathrm{L}^{\prime}$-unifier of $\Gamma$. But $\sigma(\Gamma) \vdash_{\mathrm{L}^{\prime}} \sigma(\varphi)$ and therefore $\vdash_{\mathrm{L}^{\prime}} \sigma(\varphi)$. Now consider any homomorphism $e: \mathbf{T}_{\mathcal{L}} \rightarrow \mathbf{A}$. Since $h$ is a surjective homomorphism from $\mathbf{B}$ to $\mathbf{A}$, there exists a homomorphism $k: \mathbf{A} \rightarrow \mathbf{B}$ such that $h \circ k$ is the identity map on $\mathbf{A}$. But $\vdash_{L^{\prime}} \sigma(\varphi)$ and hence $k \circ e \circ \sigma(\varphi) \in D_{B}$. Therefore $e \circ \sigma(\varphi)=h \circ k \circ e \circ \sigma(\varphi) \in h\left[D_{B}\right] \subseteq D_{A}$. So $\vdash_{\mathrm{L}} \sigma(\varphi)$.

Example 6.3. The 3-valued Łukasiewicz logic $\mathrm{E}_{3}$ and Jaśkowski logic $\mathrm{J}_{3}$ may both be presented using the 3-element Wajsberg algebra $\mathbf{L}_{\mathbf{3}}$ (Example 4.11) but with 1 as designated value for $\mathrm{E}_{3}$ and $\frac{1}{2}$ and 1 as designated values for $\mathrm{J}_{3}$. That is, $\mathrm{E}_{3}=\left(\mathbf{L}_{\mathbf{3}},\{1\}\right)$ and $\mathrm{J}_{3}=$ $\left(\mathbf{L}_{\mathbf{3}},\left\{\frac{1}{2}, 1\right\}\right)$. In this case, there is a smallest subalgebra of $\mathbf{F}_{\mathbf{L}_{\mathbf{3}}}(\omega)$ isomorphic to $\mathbf{L}_{\mathbf{3}} \times \mathbf{L}_{\mathbf{2}}$ with a homomorphism that maps $\mathbf{L}_{\mathbf{3}} \times \mathbf{L}_{\mathbf{2}}$ onto $\mathbf{L}_{\mathbf{3}}$ and sends the inherited designated values $(1,1)$ to 1 , and $\left(\frac{1}{2}, 1\right)$ to $\frac{1}{2}$. We therefore obtain a logic $\left(\mathbf{L}_{\mathbf{3}} \times \mathbf{L}_{\mathbf{2}},\{(1,1)\}\right)$ corresponding to admissibility in $\mathrm{E}_{3}$, and another logic $\left(\mathbf{L}_{\mathbf{3}} \times \mathbf{L}_{\mathbf{2}},\left\{\left(\frac{1}{2}, 1\right),(1,1)\right\}\right)$ corresponding to admissibility in $\mathrm{J}_{3}$.

\section{Concluding Remarks}

The algorithms described in this paper have been implemented in the tool TAFA, a Delphi XE2 implementation compiled for Windows operating systems available for download online at https://sites.google.com/site/admissibility. This tool has been used in particular to obtain minimal generating sets and to check structural completeness and almost structural completeness for all 3-element groupoids (i.e., 3-element algebras with a single binary operation) of which there are 3330 up to isomorphism. The cardinalities of the minimal free algebras required to check admissibility range from 3 to 1296, while the minimal generating algebras have at most 9 elements. 2676 of the groupoids are structurally complete and a further 254 are almost structurally complete. For further details of TAFA and these experiments, consult the system description [34].

Although the algorithms described here produce minimal sets of algebras for testing admissibility (and of course have to be run only once for a given finite set of finite algebras), the steps involved - generating a suitable free algebra and its subalgebras and checking homomorphic images - may be computationally unfeasible. For this reason, our case studies have consisted so far mostly of algebras with 5 or fewer elements where the appropriate free algebra is of a reasonable size (less than 1000 elements, say). This bottleneck could perhaps be addressed by introducing heuristics such as constructing and checking small subalgebras of free algebras incrementally, rather than beginning with the whole free algebra, or calculating only some and not all of the congruences in the MinGENSET algorithm. We might also hope to obtain faster algorithms for cases where the given algebras admit certain properties such as congruence distributivity or permutability. 
We recall finally that one of the motivations for investigating admissibility in arbitrary finite algebras or, similarly, finite-valued logics, is to obtain admissible quasiequations or rules that can be used to improve proof systems (shortening derivations, constraining proof search, simplifying rules, etc.) for these algebras or logics. The work reported here provides a significant step towards this goal. The next, equally ambitious, step will be to determine which rules have the potential to be useful for a given finite algebra or finite-valued logic and to add these appropriately to corresponding proof systems.

\section{REFERENCES}

[1] F. Baader and B. Morawska. Unification in the description logic EL. Log. Methods Comput. Sci., 6(3), 2010.

[2] M. Baaz, C. G. Fermüller, and G. Salzer. Automated deduction for many-valued logics. In Handbook of Automated Reasoning, volume II, chapter 20, pages 1355-1402. Elsevier, 2001.

[3] S. Babenyshev and V. Rybakov. Linear temporal logic LTL: Basis for admissible rules. J. Log. Comput., 21(2):157-177, 2011.

[4] S. Babenyshev and V. Rybakov. Unification in linear temporal logic LTL. Ann. Pure Appl. Logic, 162(12):991-1000, 2011.

[5] S. Babenyshev, V. Rybakov, R. A. Schmidt, and D. Tishkovsky. A tableau method for checking rule admissibility in S4. In Proc. M4M 2009, volume 262 of ENTCS, pages 17-32, 2010.

[6] C. Bergman. Structural completeness in algebra and logic. In Algebraic Logic, volume 54 of Colloq. Math. Soc. János Bolyai, pages 59-73. North-Holland, Amsterdam, 1991.

[7] C. Bergman and G. Slutzki. Complexity of some problems concerning varieties and quasi-varieties of algebras. SIAM J. Comput., 30(2):359-382, 2000.

[8] G. Birkhoff. On the structure of abstract algebras. Proc. Camb. Philos. Soc., 31:433-454, 1935.

[9] G. Birkhoff. Lattice Theory. Amer. Math. Soc., New York, 1940.

[10] S. Burris and H. P. Sankappanavar. A Course in Universal Algebra, volume 78 of Graduate Texts in Mathematics. Springer, New York, 1981.

[11] L. M. Cabrer and G. Metcalfe. Admissibility via natural dualities. Manuscript.

[12] X. Caicedo. The subdirect decomposition theorem for classes of structures closed under direct limits. J. Austral. Math. Soc. Ser. A, 30(2):171-179, 1980/81.

[13] P. Cintula and G. Metcalfe. Structural completeness in fuzzy logics. Notre Dame J. Form. Log., 50(2):153-183, 2009.

[14] P. Cintula and G. Metcalfe. Admissible rules in the implication-negation fragment of intuitionistic logic. Ann. Pure Appl. Logic, 162(10):162-171, 2010.

[15] J. Demel. Fast algorithms for finding a subdirect decomposition and interesting congruences of finite algebras. Kybernetika, 18:121-130, 1982.

[16] N. Dershowitz and Z. Manna. Proving termination with multiset orderings. Commun. ACM, 22:465-476, 1979.

[17] S. Ghilardi. Unification in intuitionistic logic. J. Symbolic Logic, 64(2):859-880, 1999.

[18] S. Ghilardi. Best solving modal equations. Ann. Pure Appl. Logic, 102(3):184-198, 2000.

[19] S. Ghilardi. A resolution/tableaux algorithm for projective approximations in IPC. Log. J. IGPL, 10(3):227-241, 2002.

[20] V. A. Gorbunov. Algebraic Theory of Quasivarieties. Springer, 1998.

[21] R. Hähnle. Automated Deduction in Multiple-Valued Logics. Oxford Univ. Press, 1993.

[22] R. Iemhoff. On the admissible rules of intuitionistic propositional logic. J. Symbolic Logic, 66(1):281-294, 2001.

[23] R. Iemhoff and G. Metcalfe. Hypersequent systems for the admissible rules of modal and intermediate logics. In Proc. LFCS 2009, volume 5407 of LNCS, pages 230-245. Springer, 2009.

[24] R. Iemhoff and G. Metcalfe. Proof theory for admissible rules. Ann. Pure Appl. Logic, 159(1-2):171-186, 2009.

[25] E. Jeřábek. Admissible rules of modal logics. J. Logic Comput., 15:411-431, 2005.

[26] E. Jeřábek. Admissible rules of Łukasiewicz logic. J. Logic Comput., 20(2):425-447, 2010.

[27] E. Jeřábek. Bases of admissible rules of Łukasiewicz logic. J. Logic Comput., 20(6):1149-1163, 2010. 
[28] B. Jónsson. Sublattices of a free lattice. Canad. J. Math., 13:256-264, 1961.

[29] G. Metcalfe. An Avron rule for fragments of R-mingle. J. Logic Comput., to appear.

[30] G. Metcalfe, N. Olivetti, and D. Gabbay. Proof Theory for Fuzzy Logics, volume 36 of Applied Logic. Springer, 2008.

[31] G. Metcalfe and C. Röthlisberger. Admissibility in De Morgan algebras. Soft Comput., 16(11):1875$1882,2012$.

[32] G. Metcalfe and C. Röthlisberger. Unifiability and admissibility in finite algebras. In Proc. CiE 2012, volume 7318 of $L N C S$, pages 485-495. Springer, 2012.

[33] G. L. Miller. Graph isomorphism, general remarks. J. Comput. System Sci., 18(2):128-142, 1979.

[34] C. Röthlisberger. TAFA - a tool for admissibility in finite algebras. Submitted. Software downloadable from https://sites.google.com/site/admissibility.

[35] V. Rybakov. Admissibility of Logical Inference Rules, volume 136 of Studies in Logic and the Foundations of Mathematics. Elsevier, Amsterdam, 1997.

[36] R. Zach. Proof theory of finite-valued logics. Master's thesis, Technische Universität Wien, 1993.

This work is licensed under the Creative Commons Attribution-NoDerivs License. To view a copy of this license, visit http://creativecommons.org/licenses/by-nd/2.0/ or send a letter to Creative Commons, 171 Second St, Suite 300, San Francisco, CA 94105, USA, or Eisenacher Strasse 2, 10777 Berlin, Germany 